\title{
Extending the scope of holographic mutual information and chaotic behavior
}

\section{Nilanjan Sircar, Jacob Sonnenschein and Walter Tangarife}

The Raymond and Beverly Sackler School of Physics and Astronomy, Tel Aviv University, Ramat Aviv 69978, Israel

E-mail: nilanjan.tifr@gmail.com, cobi@post.tau.ac.il, waltert@post.tau.ac.il

ABSTRACT: We extend the use of holography to investigate the scrambling properties of various physical systems. Specifically, we consider: (i) non-conformal backgrounds of black $D p$ branes, (ii) asymptotically Lifshitz black holes, and (iii) black AdS solutions of GaussBonnet gravity. We use the disruption of the entanglement entropy as a probe of the chaotic features of such systems. Our analysis shows that these theories share the same type of behavior as conformal theories as they undergo chaos; however, in the case of Gauss-Bonnet gravity, we find a stark difference in the evolution of the mutual information for negative Gauss-Bonnet coupling. This may signal an inconsistency of the latter.

KeYwords: AdS-CFT Correspondence, Gauge-gravity correspondence, p-branes

ARXIV EPRINT: 1602.07307 


\section{Contents}

1 Introduction and summary 1

2 Scrambling time in general geometries 5

3 The butterfly effect in non-conformal backgrounds $\quad 6$

3.1 Black $D p$-brane geometry

3.2 Mutual information in black $D p$-brane geometries 8

$\begin{array}{ll}3.3 \text { Shock wave in black } D p \text { branes and scrambling time } & 10\end{array}$

$\begin{array}{ll}3.4 & \text { Extremal surfaces for half plane in TFD } \\ \end{array}$

3.5 Mutual Information in shock wave black $D p$ brane 13

4 A black hole in asymptotically Lifshitz solutions $\quad 14$

$\begin{array}{lll}4.1 \text { Bulk metric } & 14\end{array}$

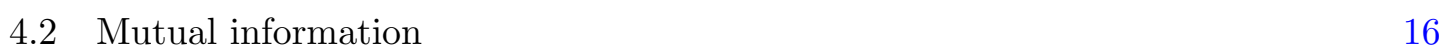

$\begin{array}{ll}\text { 4.3 Shock wave in Lifshitz black hole and scrambling time } & 16\end{array}$

$\begin{array}{lll}4.4 & \text { Extremal surfaces for half plane in TFD } & 17\end{array}$

4.5 Mutual information in shock wave Lifshitz Black Hole 18

5 Gauss-Bonnet gravity 18

$\begin{array}{lll}5.1 & \text { Black hole geometry in Gauss-Bonnet gravity } & 18\end{array}$

5.2 Mutual information in Gauss-Bonnet gravity 20

$\begin{array}{lll}5.3 & \text { Shock wave geometry and scrambling time } & 21\end{array}$

5.4 Disruption of mutual information after the shock wave 22

6 Outlook 24

A A series expansion of EE in Dp-brane backgrounds $\quad 26$

B Two sided entanglement entropy for half plane in the case of D5 brane 28

\section{Introduction and summary}

In recent years, there has been an increasing convergence of interests among the quantum information, high energy theory and condensed matter communities. A common ground of this interdisciplinary interaction is the use of entanglement entropy (EE) to characterize quantum systems. In the gauge/gravity correspondence, the entanglement entropy of a boundary region $A$ is determined by the area of the minimized codimension- 2 bulk surface that coincides with the entangling surface at the boundary [1-3]. An extra ingredient is the 
association of the properties of a black hole in the bulk with a thermal state in the boundary theory, providing a powerful tool to answer questions regarding the thermalization of strongly coupled theories.

Equilibration in thermal systems has been studied in holography in a variety of settings via EE. Some of the most relevant approaches include calculating quasi-normal modes of small perturbations in the bulk geometry to determine the relaxation rate of the perturbation (near equilibrium) [4] and following the formation of a black hole in the bulk (far from equilibrium) [5]. The latter approach has been used to further understand the time evolution of entanglement entropy and mutual information as a system thermalizes [5-13].

Black holes are conjectured to be fast scramblers, meaning that they thermalize faster than other systems in nature $[14,15]$. Specifically, the so-called scrambling time scales logarithmically with the black hole entropy,

$$
t_{*} \sim \frac{\beta}{2 \pi} \log S_{\mathrm{BH}} .
$$

This is the time scale at which all correlations in a system have been destroyed after introducing a perturbation. The scrambling time plays a crucial role in the evolution entanglement entropy. In [16], this was illustrated by looking at the time evolution of the two-dimensional thermo-field double state (TFD)

$$
|\Psi\rangle \equiv \sum_{i} e^{-\beta E_{i} / 2}|i\rangle_{L} \otimes|i\rangle_{R}
$$

where $|i\rangle_{L}$ and $|i\rangle_{R}$ are identical states of two quantum theories. The holographic dual of such a pure state is given by the eternal AdS black hole in $2+1$ dimensions [17]. A perturbation to this state is introduced by adding a shock wave, with energy $E$ at a boundary time $t_{w}$, to the two-sided AdS black hole geometry. One can then characterize the dependence between the two boundaries by computing mutual information, ${ }^{1}$

$$
I(A, B) \equiv S_{A}+S_{B}-S_{A \cup B},
$$

where $A$ and $B$ are space-like regions in each boundary. In the specific case of a $2+1$ dimensional bulk, $S_{A}$ and $S_{B}$ are given by the length of the geodesic that extends between the boundary of the intervals $A$ and $B$, respectively. The geodesic used to compute $S_{A \cup B}$ is one traversing across the black hole horizon, joining the two boundaries. For large intervals $\left(\sinh \left(\frac{\pi \phi \ell}{\beta}\right)>1, \phi<\pi\right)$, the resultant mutual information is given by

$$
I(A, B)=\frac{\ell}{G}\left(\log \sinh \frac{\pi \phi \ell}{\beta}-\log \left(1+\frac{E \beta}{4 S} e^{2 \pi t_{w} / \beta}\right)\right),
$$

where $\phi$ is the angular size of the $A$ and $B, \ell$ is the AdS radius, and $S$ is the black hole entropy. From this expression, it can be seen $I(A, B)$ vanishes when (for $\beta \gg \ell$ ),

$$
t_{w}=t_{*}=\frac{\phi \ell}{2}+\frac{\beta}{2 \pi} \log \frac{2 S}{\beta E} .
$$

\footnotetext{
${ }^{1}$ The mutual information between subsystems in two different CFTs of the thermofield double was introduced and studied in [18] and coined as thermo-mutual information.
} 
This disruption of the entanglement, which corresponds to the saturation of mutual information, is evidence of the state's high sensitivity to variations of initial conditions, a phenomenon known as the butterfly effect. Because mutual information acts as an upper bound on the correlations between the subsystems, the scrambling time defined in this fashion sets the time at which any sort of dependence between the $A$ and $B$ has been erased [19-21]. This analysis has been generalized to $A d S_{d}$ in [22], multiple shock waves [23], and localized shock waves [20, 24]. Likewise, the stringy corrections of the scrambling time were presented in [25].

In a follow up work, a bound on how fast a thermal quantum field theory (QFT) can develop chaos was conjectured in [26]. Based on the results from Einstein gravity calculations [16], a bound on the largest Lyapunov exponent was proposed $\lambda_{L} \leq \frac{2 \pi k_{B} T}{\hbar}$. $^{2}$

In this article, we extend the scope of the use of holography to investigate the chaotic behavior, and in particular the scrambling time, of various systems which have not yet been explored: (i) The non-conformal backgrounds of black Dp branes [28]. (ii) Black holes of asymptotically Lifshitz non-relativistic backgrounds [29] (iii) Conformal backgrounds which are solutions of the Gauss-Bonnet gravity [30-34].

Our analysis is based on the idea, suggested in [16], of using the disruption of the entanglement entropy as the probe of chaos. We apply the procedure and techniques presented in [22] on the three classes of background mentioned above. The procedure includes the following steps: (i) We compute the entanglement entropy $S_{A}$ and $S_{B}$ associated with a strip of width $L$. This includes the renormalization of the EE's by subtracting the divergent parts of them. (ii) We compute the mutual information $I(A, B)$. (iii) We introduce a null perturbation in the form of a shock wave and determine the corresponding backreacted geometry. (iv) We re-express the metric in Kruskal coordinates $u$ and $v$. The shock wave propagates along a constant $u$ and causes a shift in $v$ denoted by $\alpha$ (see (2.8)). We compute $\alpha$ and get an approximation of the scrambling time $t_{*}$ from the condition of $\alpha \sim 1$. (v) We compute the dependence of $\alpha$ on $r_{0}$, the value of the radial coordinate of the tip of the entangling surface, and then obtain the mutual information $I\left(A_{h}, B_{h}\right)$ associated with the shock wave as a function of $\alpha$; in particular, we obtain numerically $\alpha_{*}$, where $I$ vanishes. For the cases of the $D p$-brane and Lifshitz backgrounds, we determine EE using the prescription of [1,3] whereas for the GB gravity we adopt the prescription of [32] and [33].

The outcome of our holographic analysis is the following:

- The dual gravitational description of Yang Mills theories in $p+1$ dimensions with 16 supersymmetries in terms of the $D p$ backgrounds is reliable only in range of values of the effective coupling where the corresponding curvature and string coupling are small [28]. Our analysis of the mutual information in this range shows that the behavior for $p \leq 4, p \neq 3$ the non-conformal cases is similar to that of $p=3$ the conformal case. We found that the dependence of $I$ on $\alpha$ becomes steeper upon increasing $p$ for $p<5$. For $p=5$ the situation is just the opposite and the dependence is flatter. The fact that for $p \geq 5$ the dependence is different is consistent with the findings of [35] and [36].

\footnotetext{
${ }^{2}$ The Lyapunov exponent was associated to the damping coefficient of the thermal gluon plasma using holography in [27].
} 
- For the Lifshitz non-relativistic backgrounds that are characterized by a different scaling of the time and space directions, we found that basically the behavior is like for the relativistically invariant backgrounds. The dependence of $\alpha$ on the width of the interval $L$ is steeper as one increases $z$ and the dependence of $I$ on $\alpha$ is flatter, namely, $I$ vanishes at a larger values of $\alpha$

- In the GB gravity, for positive coupling $\lambda$ which is in accordance with causality [37], the dependence of the mutual information in the presence of a shock wave on $\alpha$ is smooth and monotonically decreasing, in a similar manner to that of the usual Einstein gravity case. $\alpha_{*}$, where the mutual information vanishes increases with the increase of $\lambda$. On the other hand for negative $\lambda$ and in the allowed region according to [37] we encountered an anomalous "cusp" behavior depicted in figure 14. This follows from the fact that $z_{0}$ is a double valued function of $\alpha$, namely, for any given value of $\alpha$ there are two values of $z_{0}$ and hence two values of the mutual information $I$. Note that whereas around $z_{0}$ that hits the singularity the curvature is large, this is not the case for the point of the "cusp". This type of anomalous behavior was detected also in the computation of the entanglement entropy in [38]. It does not seem likely that it signals a special behavior in the dual boundary field theory but rather that the GB gravity maybe inconsistent. An inconsistency with causality was found in [39] though there the causal behavior was found out to for both positive and negative values of $\lambda$ and in our case it is only for the latter.

The paper is organized as follows: in the next section we describe the determination of the scrambling time in general. Section 3 is devoted to the analysis of the chaotic behavior associated with non-conformal backgrounds of the form of Dp branes. In section 3.1 the geometry of black Dp branes is reviewed. We then compute the mutual information in these geometries in section 3.2. In section 3.3 we write down the relation between $\alpha$ and $t_{*}$ and determine the latter. The Penrose diagram associated with the minimal surface in the presence of the shock wave is drawn in section 3.4. We then compute the relation between $\alpha$ and $r_{0}$. We then determine the mutual information as a function of $\alpha$ in section 3.5. Section 4 is devoted to the case of asymptotically Lifshitz non-relativistic background. In section 4.1 we review the basic properties of the Lifshitz backgrounds and write down an action of a particular model and its corresponding metric. The mutual information of this system prior to introducing the shock wave is written down in section 4.2 . In section 4.3 we introduce the shock wave and approximate the scrambling time. The description of the extremal surfaces for half plane in TFD is given in section 4.4 and the calculation of the mutual information in section 4.5. In section 5 we present the analysis of the holographic mutual information in the case of Gauss-Bonnet higher curvature gravity. In section 5.1 we present the action and the corresponding metric solution of the equations of motion. The mutual information, scrambling time and the extremal surface are analyzed in subsections 5.2, 5.3 and 5.4 respectively. In section 6 we summarize the results of this paper and list several open questions. We then add in appendix A a series expansion of EE in Dp-brane backgrounds. In appendix B we give an analytic expression for two sided entanglement etropy for half planes in case of $D 5$ brane. 


\section{Scrambling time in general geometries}

In this section, we summarize the generalities of the scrambling time calculation in an eternal black hole geometry. We follow the analysis of a generic metric presented in the appendix of [16].

Let us start by considering a $d+1$-dimensional metric of the form,

$$
\begin{aligned}
d s^{2} & =-f(r) d t^{2}+\frac{d r^{2}}{f(r)}+d \Sigma_{d-1}^{2}, \\
d \Sigma_{d-1}^{2} & =g_{i j}\left(r, x^{i}\right) d x^{i} d x^{j} .
\end{aligned}
$$

We further assume the existence of a non-extremal horizon at $r=r_{h}$, such that $f\left(r_{h}\right)=0$ and $f^{\prime}\left(r_{h}\right) \neq 0$. The inverse Hawking temperature associated with the black hole is given as $\beta=\frac{4 \pi}{f^{\prime}\left(r_{h}\right)}$.

We then rewrite the metric using the Kruskal coordinates,

$$
\begin{aligned}
u v & =-e^{f^{\prime}\left(r_{h}\right) r_{*}(r)}, \quad u / v=e^{-f^{\prime}\left(r_{h}\right) t}, \\
d s^{2} & =-\frac{4 f(r)}{f^{\prime}\left(r_{h}\right)^{2}} e^{-f^{\prime}\left(r_{h}\right) r_{*}(r)} d u d v+d \Sigma_{d-1}^{2},
\end{aligned}
$$

where $d r_{*}=\frac{d r}{f(r)}$ is the so-called tortoise coordinate, which behaves such that $r_{*} \rightarrow-\infty$ as $r \rightarrow r_{h}$.

We now add a global null perturbation of asymptotic energy $E \ll M$ ( $M$ is the ADM mass) at time $t_{w}$ and radius $r=\Lambda . \tilde{u}, \tilde{v}$ denotes coordinates to the left of the perturbation and $u, v$ to the right. The shell propagates on a $u=$ const surface given by,

$$
\tilde{u}_{w}=e^{\frac{\tilde{f}^{\prime}\left(\tilde{r}_{h}\right)}{2}\left(\tilde{r}_{*}(\Lambda)-t_{w}\right)} \quad u_{w}=e^{\frac{f^{\prime}\left(r_{h}\right)}{2}\left(r_{*}(\Lambda)-t_{w}\right)} .
$$

We patch the two sides of the geometry along this surface, using the following matching condition,

$$
\tilde{u}_{w} \tilde{v}=-e^{\tilde{f}^{\prime}\left(\tilde{r}_{h}\right) r \tilde{r}_{*}(\tilde{r})} \quad u_{w} v=-e^{f^{\prime}\left(r_{h}\right) r_{*}(r)} .
$$

We expect that at large $t_{w}$ and order $\mathcal{O}(E)$

$$
\tilde{v}=v+\alpha .
$$

The Penrose diagram corresponding to the backreacted geometry is presented in figure 1 .

For small $E$ we can approximate $\tilde{u}_{w}=u_{w}$. Large $t_{w}$ pushes $r$ to $r_{h}$, so that we can approximate $f(r)=f^{\prime}\left(r_{h}\right)\left(r-r_{h}\right)+\cdots$. Then $e^{f^{\prime}\left(r_{h}\right) r_{*}}=\left(r-r_{h}\right) C\left(r, r_{h}\right)$, where $C\left(r, r_{h}\right)$ is smooth and non-zero at $r=r_{h}$. Then, to linear order in $E$,

$$
\alpha=\left.\frac{E}{u_{w}} \frac{d}{d M}\left[\left(r_{h}-r\right) C\left(r, r_{h}\right)\right]\right|_{r=r_{h}}=\frac{E}{u_{w}} \frac{d r_{h}}{d M} C\left(r_{h}, r_{h}\right) .
$$

By Bekenstein-Hawking area formula we can relate $r_{h}$ and the entropy, ${ }^{3}$

$$
S_{\mathrm{BH}}=\frac{V_{d-1}\left(r_{h}\right)}{4 G_{N}},
$$

\footnotetext{
${ }^{3}$ This result is valid also for higher derivative black holes with planar horizon [30].
} 


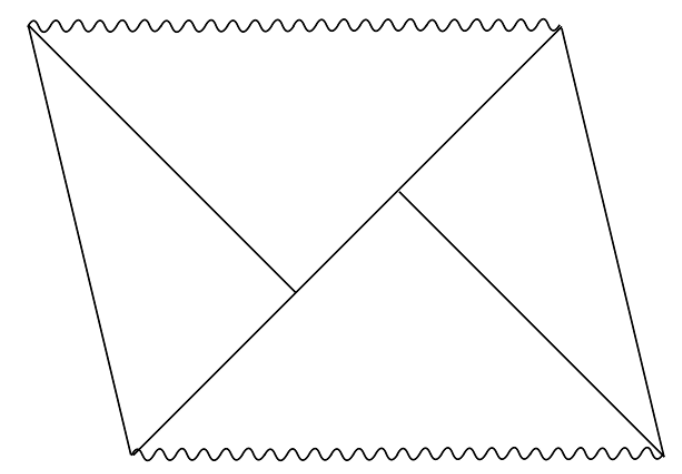

Figure 1. The geometry of an eternal black hole under the perturbation of a shock wave.

where, $V_{d-1}\left(r_{h}\right)=\int_{\Sigma} d^{d-1} x \sqrt{g_{\Sigma}}$ is the area of the horizon. Now we can use the first law,

$$
E=\delta M=T \delta S=T \frac{V_{d-1}^{\prime}\left(r_{h}\right)}{V_{d-1}\left(r_{h}\right)} S_{\mathrm{BH}} \frac{d r_{h}}{d M} E
$$

which gives,

$$
\frac{d r_{h}}{d M}=\frac{1}{T} \frac{V_{d-1}\left(r_{h}\right)}{V_{d-1}^{\prime}\left(r_{h}\right)} \frac{1}{S_{\mathrm{BH}}}
$$

then,

$$
\alpha=\frac{E}{T} C\left(r_{h}, r_{h}\right) \frac{V_{d-1}\left(r_{h}\right)}{V_{d-1}^{\prime}\left(r_{h}\right)} \frac{1}{S_{\mathrm{BH}}} e^{-\frac{2 \pi}{\beta}\left(r_{*}(\Lambda)-t_{w}\right)} .
$$

The scrambling time is given by $t_{w}=t_{*}$ when $\alpha \sim 1$ [16]. Inverting that,

$$
t_{*}=r_{*}(\Lambda)+\frac{\beta}{2 \pi} \log \left[\frac{V_{d-1}^{\prime}\left(r_{h}\right)}{V_{d-1}\left(r_{h}\right)} \frac{T}{E} \frac{1}{C\left(r_{h}, r_{h}\right)} S_{\mathrm{BH}}\right] .
$$

It is noteworthy that although the coordinate $r_{*}$ can be shifted by a arbitrary constant, $t_{*}$ is not changed by it, as the combination $r_{*}(\Lambda)-\frac{\beta}{2 \pi} \log C\left(r_{h}, r_{h}\right)$ is invariant under this shift. Let us define two dimensionless constant $c_{1}, c_{2}$ depending on the details of the geometry (as illustrated in following sections),

$$
\frac{V_{d-1}^{\prime}\left(r_{h}\right)}{V_{d-1}\left(r_{h}\right)}=\frac{c_{1}}{r_{h}}, \quad \text { and } \quad C\left(r_{h}, r_{h}\right)=\frac{c_{2}}{r_{h}} .
$$

For shock wave energy (density in case of non compact horizons) $E \sim T$,

$$
t_{*}=r_{*}(\Lambda)+\frac{\beta}{2 \pi} \log S_{\mathrm{BH}}+\frac{\beta}{2 \pi} \log \frac{c_{1}}{c_{2}} .
$$

Notice that $S_{\mathrm{BH}}$ denotes entropy density in case of non-compact horizons.

\section{The butterfly effect in non-conformal backgrounds}

In this section, we want to study the disruption of mutual information in non-conformal theories in $p+1$ dimensions. These are theories that correspond to black $p$-brane supergravity solutions [28]. We begin by describing the bulk metric in the Einstein frame and 
computing the mutual information for the unperturbed geometry. We then proceed to find the scrambling time after adding a shock wave perturbation to the two-sided black hole, and conclude by numerically verifying the disruption of mutual information between the two boundary theories.

\subsection{Black $D p$-brane geometry}

We consider the near extremal black $p$-branes whose geometry is given by [28]

$$
d s^{2}=-f(r) d t^{2}+\frac{d r^{2}}{f(r)}+\left(\frac{r}{R_{p}}\right)^{\frac{7-p}{2}} d \vec{y}^{2}+R_{p}^{\frac{7-p}{2}} r^{\frac{3-p}{2}} d \Omega_{8-p}^{2}
$$

where

$$
f(r) \equiv\left(\frac{r}{R_{p}}\right)^{\frac{7-p}{2}}\left(1-\left(\frac{r_{H}}{r}\right)^{7-p}\right), \quad R_{p}^{7-p}=(4 \pi)^{\frac{5-p}{2}} \Gamma\left(\frac{7-p}{2}\right) g_{s} l_{s}^{7-p} N
$$

and the dilaton field is

$$
e^{\phi-\phi_{\infty}}=\left(\frac{R_{p}}{r}\right)^{\frac{(7-p)(3-p)}{4}}, \quad g_{s}=e^{\phi_{\infty}} .
$$

This background is dual to super-Yang-Mills in $p+1$ dimensions. The non-trivial dilaton corresponds to the running of the coupling constant $g_{\mathrm{eff}}^{2}=g_{Y M}^{2} N \frac{r^{p-3}}{l_{s}^{2(p-3)}}$, where $g_{Y M}^{2}=$ $(2 \pi)^{p-2} g_{s} l_{s}^{p-3}$. The validity of the supergravity solution requires,

$$
1 \ll g_{\text {eff }}^{2} \ll N^{\frac{4}{7-p}},
$$

such that both the dilaton and the curvature are small.

For convenience, we will use a coordinate re-parametrization of the Einstein frame in our calculations. The Einstein frame metric is obtained by the following transformation of the metric in $9+1$ dimensions,

$$
g_{\mu \nu}^{E}=e^{-\frac{\phi-\phi_{\infty}}{2}} g_{\mu \nu}^{S}
$$

Then, the resultant metric is given by

$$
d s_{E}^{2}=-g_{1}(r) d t^{2}+\frac{d r^{2}}{g_{2}(r)}+\left(\frac{r}{R_{p}}\right)^{\frac{(7-p)^{2}}{8}} d \vec{y}_{\|}^{2}+R_{p}^{2}\left(\frac{r}{R_{p}}\right)^{\frac{(p-3)^{2}}{8}} d \Omega_{8-p}^{2},
$$

where,

$$
\begin{aligned}
& g_{1}(r)=\left(\frac{r}{R_{p}}\right)^{\frac{(7-p)^{2}}{8}}\left(1-\left(\frac{r_{0}}{r}\right)^{7-p}\right), \\
& g_{2}(r)=\left(\frac{r}{R_{p}}\right)^{\frac{(7-p)(p+1)}{8}}\left(1-\left(\frac{r_{0}}{r}\right)^{7-p}\right) .
\end{aligned}
$$

Now, we perform the coordinate redefinition

$$
r=\frac{\rho^{n}}{n^{n} R_{p}^{n-1}}, \quad n=\frac{8}{8+(7-p)(3-p)},
$$


under which the metric in Einstein frame can be written as

$$
\begin{aligned}
& d s_{E}^{2}=-f(\rho) d t^{2}+\frac{d \rho^{2}}{f(\rho)}+\left(\frac{\rho}{n R_{p}}\right)^{\frac{n(7-p)^{2}}{8}} d \vec{y}_{\|}^{2}+R_{p}^{2}\left(\frac{\rho}{n R_{p}}\right)^{\frac{n(p-3)^{2}}{8}} d \Omega_{8-p}^{2}, \\
& f(\rho)=\left(\frac{\rho}{n R_{p}}\right)^{n \frac{(7-p)^{2}}{8}}\left(1-\left(\frac{\rho_{H}}{\rho}\right)^{n(7-p)}\right) .
\end{aligned}
$$

In this geometry, the temperature and entropy density ${ }^{4}$ are given by,

$$
\begin{aligned}
T & =\frac{(7-p)}{4 \pi R_{p}}\left(\frac{\rho_{H}}{n R_{p}}\right)^{\frac{n}{2}(5-p)}, \\
\frac{S}{\Omega_{8} V_{p}} & =c(p) \lambda^{\frac{p-3}{5-p}} N^{2} T^{\frac{9-p}{5-p}}
\end{aligned}
$$

where

$$
c(p)=\frac{2^{\frac{2(11-2 p)}{5-p}} \pi^{\frac{19-8 p+p^{2}}{2(p-5)}} \Gamma\left(\frac{7-p}{2}\right)^{\frac{7-p}{5-p}}}{(7-p)^{\frac{9-p}{5-p}}},
$$

and $\lambda=g_{Y M}^{2} N$ is the 't Hooft coupling in the dual field theory.

In the following subsections, we will use this metric with the change in notation, for convenience,

$$
r \equiv \rho, \quad r_{h} \equiv \rho_{h}, \quad \text { and } \quad \ell \equiv n R_{p}
$$

\subsection{Mutual information in black $D p$-brane geometries}

In this section, we present an overview of the mutual information between the two boundaries of an eternal black hole in the $D p$-brane background. Specifically, we compute the mutual information (1.3) between two strips $A$ and $B$ contained in the left and right side of the geometry respectively. ${ }^{5}$ To achieve this, we must compute the EE between each strip and the rest of the system $\left(S_{A}\right.$ and $\left.S_{B}\right)$, and the EE of their union $\left(S_{A \cup B}\right)$.

We begin by reviewing the calculation of the holographic EE of an interval using the background given by (3.10) with the coordinates defined in (3.14). Each interval is defined along the coordinates

$$
y \equiv y^{1} \in[-L / 2, L / 2], y^{i=2, \cdots, p-1} \in(-\infty, \infty) .
$$

The holographic prescription [40] stipulates that $S_{A}$ (or $S_{B}$ ) is given by the codimension-2 minimal surface, in the bulk, whose boundary is the same as the boundary of $A$ (or $B$ ). We parameterize this surface by $x=\left(y(r), y^{2}, \cdots, y^{p-1}, \theta^{1}, \cdots, \theta^{8-p}\right)$. Thus, $S_{A}$ is given by

$$
S_{A}=\frac{1}{4 G_{N}^{10}} \int d^{8} x \sqrt{\operatorname{Det} G_{\text {ind }}^{(8)}}
$$

\footnotetext{
${ }^{4}$ We have used the relations of 10 -dimensional Newton's constant to $g_{s}, l_{s}: 16 \pi G_{N}^{(10)}=(2 \pi)^{7} g_{s}^{2} l_{s}^{8}$.

${ }^{5}$ We will also refer to these strips as "intervals" in the rest of this article.
} 
where $G_{\text {ind }}^{(8)}$ is the induced metric on the surface, using (3.10). This yields

$$
S_{A}=\frac{\mathcal{V} \ell^{8-p}}{4 G_{N}^{10} \ell^{d-1}} \int d r\left[\left(\frac{r}{\ell}\right)^{\frac{n}{16}\left((7-p)^{2}(p-1)+(p-3)^{2}(8-p)\right)}\left(\frac{1}{f}+\left(\frac{r}{\ell}\right)^{\frac{n}{8}(7-p)^{2}} y^{\prime 2}\right)\right]^{1 / 2},
$$

where' denotes the derivative respect to $r$ and $\mathcal{V}$ is the volume in the transverse directions. The absence of $y$ in the Lagrangian allows us to write the conservation equation

$$
\begin{array}{cc}
\frac{\left(\frac{r}{\ell}\right)^{\omega / 2} y^{\prime}}{\sqrt{y^{\prime 2}+\left(\frac{r}{\ell}\right)^{-2 \xi} \frac{1}{f}}}=\left(\frac{r_{\min }}{\ell}\right)^{\omega / 2}, & 1 /\left.y^{\prime}\right|_{r_{\min }}=0, \\
\omega \equiv \frac{p n}{8}(7-p)^{2}+\frac{n}{8}(p-3)^{2}(8-p), & \xi \equiv \frac{n}{16}(7-p)^{2} .
\end{array}
$$

Solving for $y^{\prime}$ gives us the relation between the width $L$ and the turning point $r_{\min }$,

$$
L=2 \int_{r_{\min }}^{\infty} d r\left(\frac{r}{\ell}\right)^{-2 \xi} \frac{1}{\sqrt{1-\left(\frac{r_{H}}{r}\right)^{n(7-p)}} \sqrt{\left(\frac{r}{r_{\min }}\right)^{\omega}-1}} .
$$

On the other hand, plugging (3.17) into (3.16) gives us $S_{A}$ as a function of $r_{\min }$,

$$
S_{A}=2 \frac{\mathcal{V}}{4 G_{N} \ell^{d-1}} \ell^{8-p-\sigma} \int_{r_{\min }}^{\infty} d r \frac{r^{\sigma}}{\sqrt{1-\frac{r_{H}^{s}}{r^{s}}}} \frac{1}{\sqrt{1-\left(\frac{r_{\min }}{r}\right)^{\omega}}}
$$

where

$$
\sigma \equiv \frac{n}{16}(7-p)^{2}(p-2)+\frac{n}{16}(p-3)^{2}(8-p), \quad s \equiv n(7-p) .
$$

In order to take care of the divergent contribution to (3.20), we set a cutoff at $r=\Lambda$. Then, the divergent part of the entropy is

$$
S_{A, \operatorname{div}}=2 \frac{\mathcal{V}}{4 G_{N} \ell^{d-1}} \ell^{8-p-\sigma} \frac{\Lambda^{\sigma+1}}{\sigma+1},
$$

which should be subtracted from (3.20).

To perform the integrals above, it is convenient to redefine the radial coordinate

$$
u \equiv \frac{r_{\min }}{r},
$$

which leads to

$$
S_{A, \text { finite }}=2 \frac{\mathcal{V}}{4 G_{N} \ell^{d-1}} \ell^{8-p-\sigma} r_{\min }^{1+\sigma} \int_{r_{\min } / \Lambda}^{1} d u \frac{u^{-2-\sigma}}{\sqrt{1-u^{\omega}}} \frac{1}{\sqrt{1-\left(\frac{r_{H}}{r_{\min }} u\right)^{s}}}-S_{A, \mathrm{div}},
$$

and

$$
L=2 \ell^{2 \xi} r_{\min }^{1-2 \xi} \int_{0}^{1} d u u^{-2+4 \xi+\sigma} \frac{1}{\sqrt{1-u^{\omega}} \sqrt{1-\frac{r_{H}^{s}}{r_{\min }^{s}} u^{s}}} .
$$



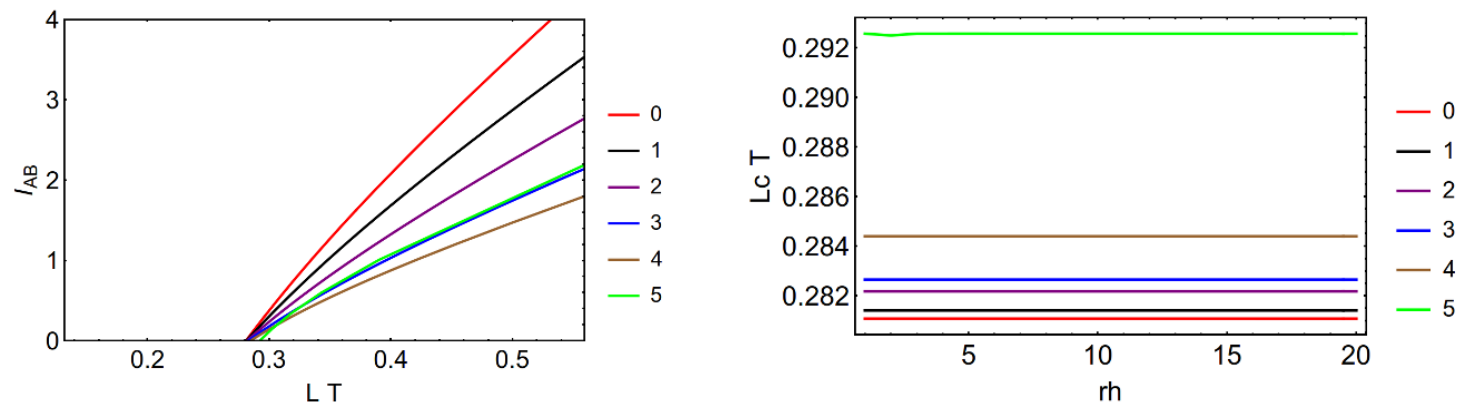

Figure 2. Left: examples of $I(A, B)$ for $r_{H} / \ell=1$, for different values of $p$. Right: critical values of $L$ for which $I(A, B)$ vanishes, in units of temperature.

In the appendix, we include some analytic expressions that approximate the result in equations (3.24) and (3.27).

Finally, we compute the area of the surface that interpolates between the two boundaries. This area corresponds to four times the area of a surface that divides the boundary in half and extends from one boundary to the black hole horizon. Defining $\tilde{u} \equiv u / r_{H}$,

$$
\begin{aligned}
S_{A \cup B, \text { finite }} & =4 \frac{\mathcal{V}}{4 G_{N} \ell^{d-1}} \ell^{8-p-\sigma} r_{H}^{\sigma+1} \int_{r_{H} / \Lambda}^{1} d \tilde{u} \frac{\tilde{u}^{-2-\sigma}}{\sqrt{1-\tilde{u}^{s}}}-2 S_{A, \mathrm{div}} \\
& =4 \frac{\mathcal{V}}{4 G_{N} \ell^{d-1}} \ell^{8-p-\sigma} r_{H}^{\sigma+1} \frac{\sqrt{\pi} \Gamma\left[-\frac{\sigma+1}{s}\right]}{s \Gamma\left[\frac{s-2 \sigma-2}{2 s}\right]}
\end{aligned}
$$

Thus, the mutual information between $A$ and $B$ is given by

$$
I(A, B)=\frac{\mathcal{V} \ell^{8-p-\sigma}}{G_{N} \ell^{d-1}} r_{\min }^{1+\sigma}\left(\int_{r_{\min } / \Lambda}^{1} d u \frac{u^{-2-\sigma}}{\sqrt{1-u^{\omega}}} \frac{1}{\sqrt{1-\left(\frac{r_{H}}{r_{\min }} u\right)^{s}}}-\int_{r_{H} / \Lambda}^{1} d \tilde{u} \frac{\tilde{u}^{-2-\sigma}}{\sqrt{1-\tilde{u}^{s}}}\right)
$$

The mutual information defined above is non-zero only when the expression in first bracket is positive. Mutual information between the regions in the two boundaries is relevant for values of $L>L_{c}$. The critical $L_{c}$, beyond which for which mutual information is non zero, as a function of the horizon $r_{H}$ is depicted in figure 2 .

\subsection{Shock wave in black $D p$ branes and scrambling time}

Let us now consider the back-reacted geometry due to a shock wave in the background of black $D p$ branes. The analysis is a specialization of that in section 2. The back-reacted geometry is given by a shift $\alpha$ in $v$ coordinate and is given by (2.12),

$$
\alpha=\frac{c_{2}}{c_{1}} \frac{E \beta}{S_{\mathrm{BH}}} e^{\frac{2 \pi}{\beta}\left(t_{w}-r_{*}(\Lambda)\right)},
$$


where,

$$
\begin{aligned}
r_{*}(\Lambda) & =\frac{2 \ell}{n(p-5)}\left(\frac{\Lambda}{\ell}\right)^{\frac{n}{2}(p-5)}, \quad p \neq 5 \\
& =\ell \log \left(\frac{\Lambda}{r_{h}}\right), \quad p=5, \\
c_{1} & =r_{h} \frac{V_{d-1}^{\prime}\left(r_{h}\right)}{V_{d-1}\left(r_{h}\right)}=\frac{\omega}{2}, \\
c_{2} & =r_{h} C\left(r_{h}, r_{h}\right)=e^{\psi\left(\frac{5-p}{2(7-p)}\right)+\gamma+\log (n(7-p))}, \quad p \neq 5 \\
& =4=c_{1}, \quad p=5 ;
\end{aligned}
$$

$\omega$ was defined in (3.18), $\psi(x)$ is the Digamma function and $\gamma$ is Euler Constant. $E$ is the energy density of the perturbation and $S_{\mathrm{BH}}$ is the black brane entropy density.

Following (2.15), the scrambling time is given by setting $\alpha=1$ and $E \sim T$, which yields

$$
t_{*}=r_{*}(\Lambda)+\frac{\beta}{2 \pi} \log S_{\mathrm{BH}}+\frac{\beta}{2 \pi} \log \frac{c_{1}}{c_{2}},
$$

where $c_{1}, c_{2}, r_{*}(\Lambda)$ are as given in previous equations. Notice that for $p<5, r_{*}(\Lambda) \rightarrow 0$ as $\Lambda \rightarrow \infty$ but for $p \geq 5, r_{*}(\Lambda)$ is divergent. We will not try to draw conclusions in the case $p=5$, in the rest of the article, as the thermodynamics or holography in general is not very well defined for $D 5$ brane [35].

\subsection{Extremal surfaces for half plane in TFD}

We now compute EE using the bulk geometry given by Einstein frame metric (3.10) in the presence of a shock wave. We consider the case where the entangling region $\left(A_{h} / B_{h}\right)$ at the left/right boundary, which is half of the space parallel to the brane and filling up the transverse direction. Then due to symmetry the minimal surface corresponding to the entangling region $A_{h} \cup B_{h}$, in the bulk, divides the space parallel to $D p$ brane in half, and area minimization is reduced to a two dimensional problem. The surface is given as $r(t)$. Then the induced metric on the bulk surface is

$$
d s_{h}^{2}=\left(-f(r)+\frac{\dot{r}^{2}}{f(r)}\right) d t^{2}+\left(\frac{r}{\ell}\right)^{\frac{n(7-p)^{2}}{8}} d \vec{y}_{\|,(p-1)}^{2}+R_{p}^{2}\left(\frac{r}{\ell}\right)^{\frac{n(p-3)^{2}}{8}} d \Omega_{8-p}^{2} .
$$

The area functional for the minimal surface is then given by

$$
\text { Area }=\mathcal{V}_{p-1} \Omega_{8-p} \frac{\ell^{8-p-m}}{n^{8-p}} \int d t r^{m} \sqrt{-f+f^{-1} \dot{r}^{2}},
$$

where, $m=\sigma+\frac{n}{16}(7-p)^{2}(\sigma$ is defined in (3.21)). The conserved quantity associated with $t$-translation symmetry,

$$
\gamma=\frac{-f r^{m}}{\sqrt{-f+f^{-1} \dot{r}^{2}}}=\sqrt{-f_{0}} r_{0}^{m}
$$

where $r_{0}$ is defined as the coordinate for which $\dot{r}=0 . r_{0}$ is assumed to be lying inside the horizon, thus, $f_{0}=f\left(r_{0}\right)$ is negative. In the limit $r_{0} \rightarrow r_{h}$, we have $\gamma \rightarrow 0$, and this should 


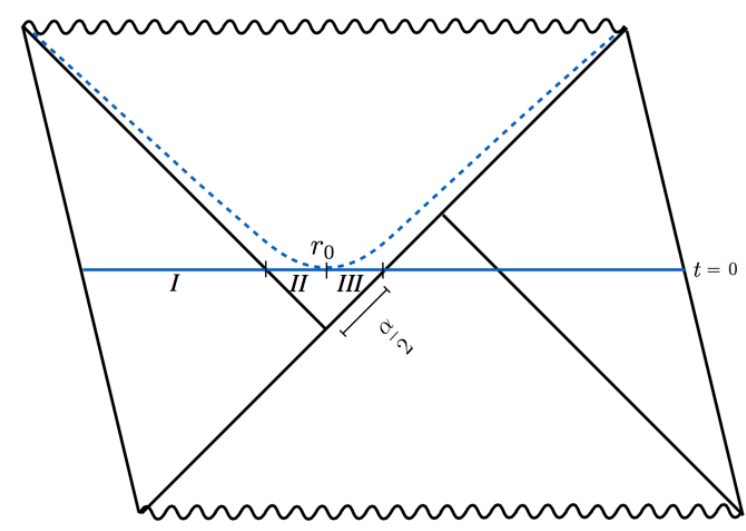

Figure 3. The minimal surface.

correspond to the limit $\alpha \rightarrow 0$ where the shock wave is absent. The time coordinate $t$ as a function of radius $r$ along the extremal curve is given by,

$$
t(r)=\int \frac{d r}{f \sqrt{1+\gamma^{-2} f r^{2 m}}} .
$$

The entanglement entropy is given by $[2,40]$,

$$
S_{A_{h} \cup B_{h}}=\frac{1}{4 G_{N}} \mathcal{V}_{p-1} \Omega_{8-p} \frac{\ell^{8-p-m}}{n^{8-p}} \int d r r^{m} \frac{1}{\sqrt{f+\gamma^{2} r^{-2 m}}}
$$

Let us divide the minimal surface of the left half in three parts, as in figure 3 . The first segment goes from boundary at $\left(u_{\Lambda},-u_{\Lambda}\right)$ to $v=0$ ( at some value of $u$ ), the second from $v=0$ to $r=r_{0}$ (at some value of $t$ ), and third from $r=r_{0}$ to $u=0$. We can now compute the area of each segment, and multiply the answer by two to get the total area. The second and third segments $\left(r=r_{h}\right.$ to $r=r_{0}$ and back) manifestly have same area. So the total area is given by twice the area of the first segment and 4 times the area of the second segment. The entanglement entropy/ area of the minimal surface is a function of $r_{0}$, which can be related to $\alpha$ as explained in the next paragraph.

Following a similar analysis as that in [22], we obtain a relation between $\alpha$ and $r_{0}$ (see eq. (36) in [22]),

$$
\alpha=2 \frac{\bar{u} \bar{v}}{u_{\Lambda}} \exp \left(K_{1}+K_{2}+K_{3}\right)\left(r_{0}\right),
$$

where,

$$
\begin{aligned}
& K_{1}=\frac{4 \pi}{\beta} \int_{\bar{r}}^{r_{0}} \frac{d r}{f}, \\
& K_{2}=\frac{2 \pi}{\beta} \int_{r_{h}}^{r_{\Lambda}} \frac{d r}{f}\left(1-\frac{1}{\sqrt{1+\gamma^{-2} f r^{2 m}}}\right), \\
& K_{3}=\frac{4 \pi}{\beta} \int_{r_{0}}^{r_{h}} \frac{d r}{f}\left(1-\frac{1}{\sqrt{1+\gamma^{-2} f r^{2 m}}}\right),
\end{aligned}
$$




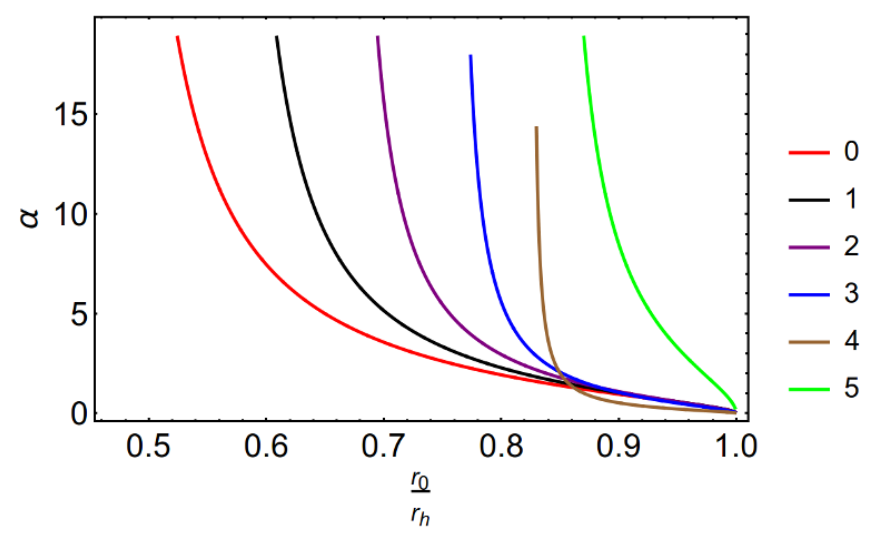

Figure 4. $\alpha$ as a function of $\frac{r_{0}}{r_{h}}$ at any temperature.

where $(\bar{u}, \bar{v})$ is a reference point in the interior of the black brane i.e. at some radial coordinate $r=\bar{r}<r_{h}$. If we choose $r_{*}(\bar{r})=0$, then $\bar{u} \bar{v}=1$ [22]. Figure 4 shows numerical plots of dependence of $\alpha$ on $\frac{r_{0}}{r_{h}}$ for various $p$. We can show that this behavior is independent of temperature for $\frac{\Lambda}{r_{h}} \gg 1$.

We can now use the relation between $\alpha$ and $r_{0}$, to get EE as a function of $\alpha$. The resultant EE has divergent contributions that are $\alpha$-independent. We can define a renormalized entanglement entropy as,

$$
S_{A_{h} \cup B_{h}}^{r e n}(\alpha)=S_{A_{h} \cup B_{h}}(\alpha)-S_{A_{h} \cup B_{h}}(0) .
$$

These calculations can be performed analytically for $p=5$ as given in appendix B. We use the results in appendix B to test our numerics.

\subsection{Mutual Information in shock wave black $D p$ brane}

Let us now consider the two strip-like regions $A$ and $B$ in the left/right boundary same as that considered in section 3.2. We are again interested in the mutual information for this configuration, but now with a backreacted geometry due the presence of a shock wave. $S_{A}+S_{B}$ is not affected due to the shock wave as the corresponding surface does not cross the horizon, so we can use the result in section 3.2. We can easily verify,

$$
I(A ; B)(\alpha, L)=S_{A}+S_{B}-S_{A \cup B}=I^{\mathrm{NSW}}(A ; B)(L)-2 S_{A \cup B}^{\mathrm{reg}}(\alpha),
$$

where $I^{\mathrm{NSW}}(A ; B)$ is the mutual information computed in absence of the shock wave as computed in section 3.2 and $S_{A \cup B}^{\mathrm{reg}}(\alpha)$ is the regularized entanglement entropy for half-regions as computed in section 3.4. Following the definition of mutual information, whenever the result is negative, it should be considered as zero. For a given length of the strip $L$ in units of temperature, we can define $\alpha=\alpha_{*}$ where mutual information vanishes.

The behavior of mutual information as function of $\alpha$ and $\alpha_{*}$ as a function of horizon radius is represented in figure 5. Note that we are only interested in the behavior of mutual information as a function of $\alpha$ and not the value itself, so we have neglected overall constants. From figure 5, it is clear that $\alpha_{*}$ is independent of temperature or $r_{h}$, as long 

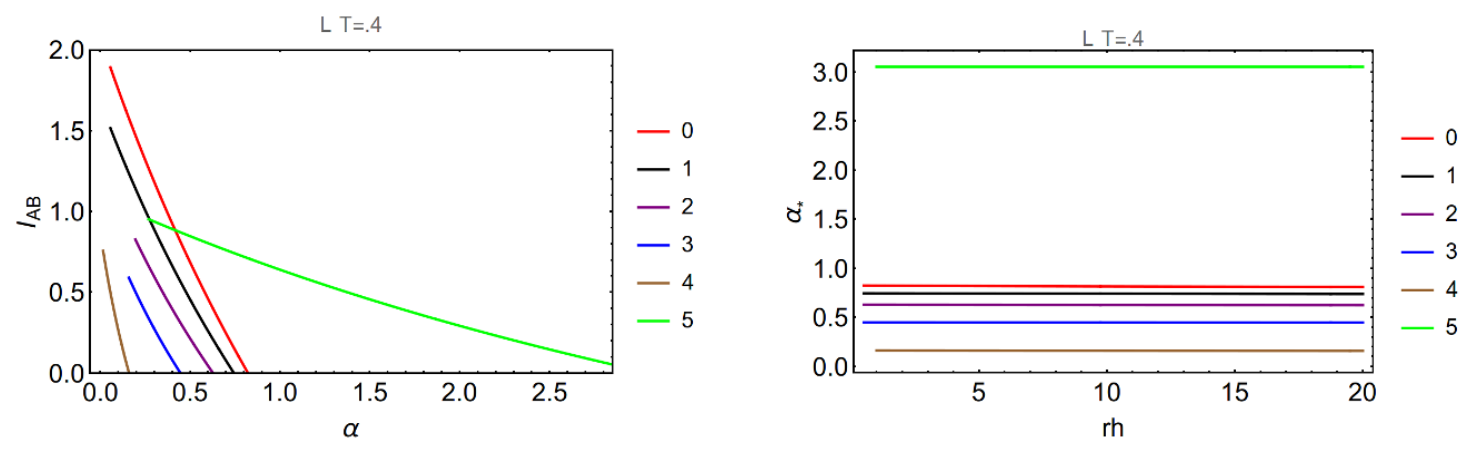

Figure 5. Left: $I(A ; B)$ as a function of $\alpha$ for various $p$ branes for $L=.4 \beta$. Right: $\alpha_{*}$ as a function of horizon radius, in units of $\ell$.

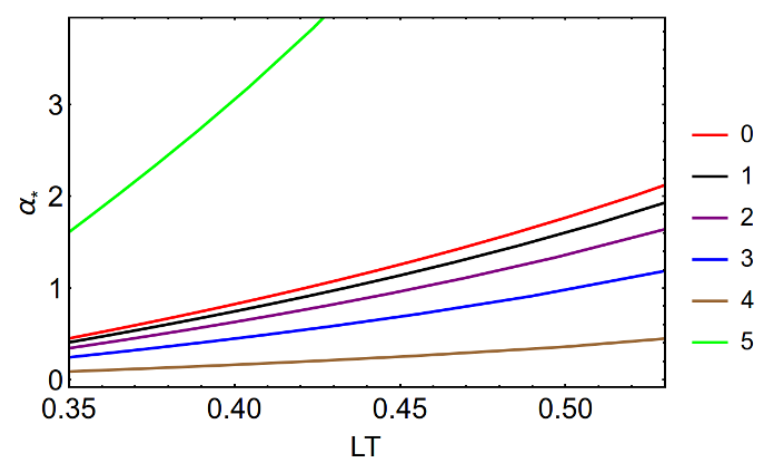

Figure 6. $\alpha_{*}$ as a function of boundary strip length in units of temperature $(L T)$.

as we keep the length of the boundary strip fixed in units of temperature. The value of $\alpha_{*}$ decreases as we increase $p$ from 0 to 4 for a given length of the boundary strip in units of temperature. The behavior for $p=5$ does not match this pattern. Figure 6 shows the behavior of $\alpha_{*}$ as a function of the length of the boundary strip in units of temperature. We now translate the behavior of $\alpha_{*}$ to that of $t_{*}$ using (3.28) (with $E \sim T$ ),

$$
t_{*}=r_{*}(\Lambda)+\frac{\beta}{2 \pi}\left(\log S_{\mathrm{BH}}(\beta, p, \lambda, N)+\log \frac{c_{1}}{c_{2}}(p)+\log \alpha_{*}\left(\frac{L}{\beta}, p\right)\right), \quad p<5 .
$$

We know from (3.13),

$$
S_{\mathrm{BH}}=N^{2} s(p, \lambda, \beta),
$$

where the function $s(p, \lambda, \beta)$ can be read off from (3.13) and is some $\mathcal{O}\left(N^{0}\right)$ number, similar to the terms $\log \frac{c_{1}}{c_{2}}(p), \log \alpha_{*}\left(\frac{L}{\beta}, p\right)$. The term $r_{*}(\Lambda)$ is small for $\Lambda \gg 1$ and $p<5$.

\section{A black hole in asymptotically Lifshitz solutions}

\subsection{Bulk metric}

In this section we shift our interest to chaos in non-relativistic field theories. We are particularly focusing on non-relativistic scale invariant field theories which arise as a fixed point description of various condensed matter systems. The scale invariance (at Lifshitz 
Fixed Point) is given by,

$$
t \rightarrow \lambda^{z} t \quad ; \quad x \rightarrow \lambda x \text { for } z \neq 1
$$

In contrast to conformal fixed points, which correspond to $z=1$. $z$ is called dynamical critical exponent. This anisotropic scaling of space and time makes these theories explicitly non-relativistic. Holographic duals of such theories were proposed in [43], which were later generalized in $[29,53]$. These dual gravity solutions are generically known as Lifshitz Solutions. For a more recent review on this topic see [54]. In particular, thermalization in these non-relativistic systems was studied holographically in $[55,56]$.

Now we look at an example of a black hole in an asymptotically Lifshitz background found in [29]. In that work, the authors consider a $2+1$ boundary. The bulk geometry is a solution to the equations of motion derived from the action

$$
S=\frac{1}{2} \int d^{4} x(R-2 \Lambda)-\int d^{4} x\left(\frac{e^{2 \Phi}}{4} F^{2}+\frac{m^{2}}{2} A^{2}+\left(e^{2 \Phi}-1\right)\right),
$$

where $A$ is a vector field and $F$ is the respective strength form. The solution to the equations of motion is given by

$$
\begin{aligned}
d s^{2} & =-f_{0}(\rho)\left(\frac{\rho}{\ell}\right)^{2 z} d t^{2}+\left(\frac{\rho}{\ell}\right)^{2} d \vec{x}^{2}+\frac{d \rho^{2}}{\left(\frac{\rho}{\ell}\right)^{2} f_{0}(\rho)}, \quad f_{0}(\rho)=1-\frac{\rho_{h}^{2}}{\rho^{2}} \\
A & =\frac{1}{\ell} f_{0}(\rho)\left(\frac{\rho}{\ell}\right)^{2} d t, \quad \Phi=-\frac{1}{2} \log \left(1-\frac{\rho_{h}^{2}}{\rho^{2}}\right) .
\end{aligned}
$$

The surface gravity of the black hole in (4.3) leads to the temperature

$$
T=\frac{\rho_{h}^{z}}{2 \pi \ell^{z+1}} .
$$

The metric (4.3) does not have the property $g_{t t} g_{\rho \rho}=-1$, which would facilitate the definition of the Kruskal coordinates as in previous examples. However, we can redefine the radial coordinate

$$
z^{2}\left(\frac{r}{\ell}\right)^{2} \equiv\left(\frac{\rho}{\ell}\right)^{2 z}
$$

which leads to the metric

$$
d s^{2}=-f(r) d t^{2}+\frac{d r^{2}}{f(r)}+\left(\frac{r}{\ell}\right)^{2 / z} d \vec{x}^{2}, \quad f(r)=z^{2}\left(\frac{r}{\ell}\right)^{2}\left(1-\left(\frac{r_{h}}{r}\right)^{\frac{2}{z}}\right),
$$

which is equipped with a temperature

$$
T=\frac{r_{h} z}{2 \pi \ell^{2}}
$$

and entropy,

$$
S_{\mathrm{BH}}=\frac{V_{2}}{4 G_{N}^{(4)}}\left(\frac{2 \pi \ell}{z}\right)^{\frac{2}{z}} T^{\frac{2}{z}} .
$$

Here we have rescaled the coordinates $\vec{x}=\left\{x_{1}, x_{2}\right\}$ by $z^{1 / z}$. 


\subsection{Mutual information}

Now we compute again the mutual information between two strips $A$ and $B$, contained in the left and right side of the geometry respectively. The strips are defined as $0<x_{1}<L$ and $0<x_{2}<\mathcal{V}(\mathcal{V} \rightarrow \infty)$. We use the metric given by (4.7).

The entanglement entropy of one strip, $S_{A}$ or $S_{B}$, with width $L$ is given by the area

$$
\text { Area }=2 \frac{\mathcal{V}}{z} \int_{r_{\min }}^{\infty} d r \frac{1}{\left(\frac{r}{\ell}\right)^{1-\frac{1}{z}} \sqrt{1-\left(\frac{r_{H}}{r}\right)^{\frac{2}{z}}}} \frac{1}{\sqrt{1-\left(\frac{r_{\min }}{r}\right)^{\frac{4}{z}}}},
$$

In order to take care of the divergent contribution to (4.10), we set a cutoff at $r=\Lambda$. Then, the divergent part of the area is

$$
\text { Area }_{\text {div }}=2 \mathcal{V} \ell\left(\frac{\Lambda}{\ell}\right)^{\frac{1}{z}}
$$

$r_{\min }$ and the width $L$ are related by

$$
L=\frac{2}{z} \int_{r_{\min }}^{\infty} d r \frac{1}{\left(\frac{r}{\ell}\right)^{1+\frac{1}{z}} \sqrt{1-\left(\frac{r_{H}}{r}\right)^{\frac{2}{z}}} \sqrt{\left(\frac{r}{r_{\min }}\right)^{\frac{4}{z}}-1}} .
$$

The finite part of the area is defined as,

$$
\text { Area }_{\text {finite }}=\text { Area }- \text { Area }_{\text {div }}
$$

Now, we compute the area of the surface that interpolates between the two boundaries. This area corresponds to four times the area of a surface that divides the boundary in half and extends from one boundary to the black hole. Defining $\tilde{u} \equiv u / r_{H}$,

$$
\text { Area }_{A \cup B, \text { finite }}=4 \frac{\mathcal{V}}{z} \int_{r_{h}}^{\infty} d r \frac{1}{\left(\frac{r}{\ell}\right)^{1+\frac{1}{z}} \sqrt{1-\left(\frac{r_{H}}{r}\right)^{\frac{2}{z}}}}-2 \text { Area }_{\text {div }},
$$

Thus, the mutual information between $A$ and $B$ is given by

$$
I(A, B) \equiv S_{A}+S_{B}-S_{A \cup B}=\frac{1}{4 G_{N}^{(4)}}\left(2 \text { Area }_{\text {finite }}-\text { Area }_{A \cup B, \text { finite }}\right) .
$$

The critical $L_{c}$, for which mutual information vanishes, as a function of the horizon $r_{H}$ is depicted in figure 7 .

\subsection{Shock wave in Lifshitz black hole and scrambling time}

Let us now consider the back-reacted geometry due to a shock wave in the background of Lifshitz black-hole. The analysis is a specialization of section 2. The back-reacted geometry is given by a shift $\alpha$ in $v$ coordinate and is given by (2.12),

$$
\alpha=\frac{c_{2}}{c_{1}} \frac{E \beta}{S_{\mathrm{BH}}} e^{\frac{2 \pi}{\beta}\left(t_{w}-r_{*}(\Lambda)\right)},
$$



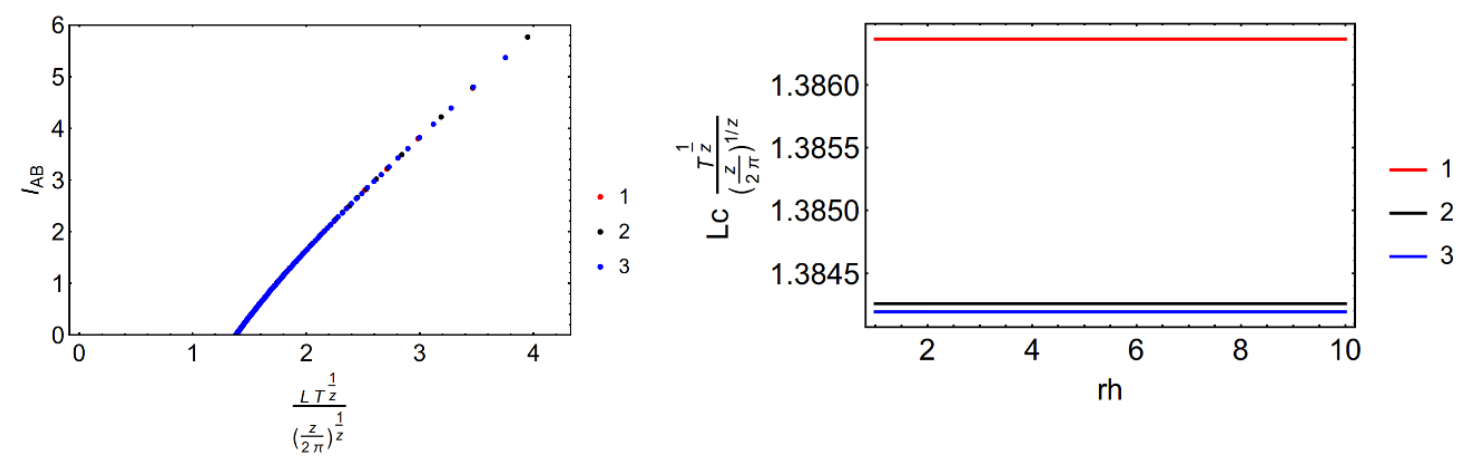

Figure 7. Left: examples of $I(A, B)$ for $r_{H} / \ell=1$, for different values of $z$. Right: critical values of $L$ for which $I(A, B)$ vanishes, in units of temperature.

where,

$$
\begin{aligned}
r_{*}(\Lambda) & =-\frac{\ell^{2}}{z^{2} \Lambda}, \\
c_{1} & =r_{h} \frac{V_{d-1}^{\prime}\left(r_{h}\right)}{V_{d-1}\left(r_{h}\right)}=\frac{1}{z}, \\
c_{2} & =r_{h} C\left(r_{h}, r_{h}\right)=\frac{2}{z} e^{\psi\left(\frac{z}{2}\right)+\gamma},
\end{aligned}
$$

where $\psi(x)$ is the Digamma function and $\gamma$ is Euler Constant.

The heuristic scrambling time is given by setting $\alpha=1$ and $E=T$ is given by (2.15),

$$
t_{*}=r_{*}(\Lambda)+\frac{\beta}{2 \pi} \log S_{\mathrm{BH}}+\frac{\beta}{2 \pi} \log \frac{c_{1}}{c_{2}},
$$

where $c_{1}, c_{2}, r_{*}(\Lambda)$ is as given in previous equations.

\subsection{Extremal surfaces for half plane in TFD}

In this section, we analyze the extremal surface that extends between the two boundaries of the geometry (4.7) in the presence of a shock wave. We consider the case where the entangling region $\left(A_{h} / B_{h}\right)$, at the left/right boundary respectively, is half of the space parallel to the brane and fills up the transverse direction. This analysis is similar to that done in section 3.4.

The time coordinate $t$ as a function of radius $r$ along the extremal curve is now given by,

$$
t(r)=\int \frac{d r}{f \sqrt{1+\gamma^{-2} f r^{2 m}}}, \quad m=\frac{1}{z},
$$

where,

$$
\gamma=\sqrt{-f\left(r_{0}\right)} r_{0}^{m}
$$

and $r=r_{0}\left(<r_{h}\right)$ is the deepest the surface penetrates the region beyond the horizon, as described in section 3.4. The entanglement entropy is given by,

$$
S_{A_{h} \cup B_{h}}=\frac{1}{4 G_{N}} \mathcal{V} \int d r r^{m} \frac{1}{\sqrt{f+\gamma^{2} r^{-2 m}}}
$$




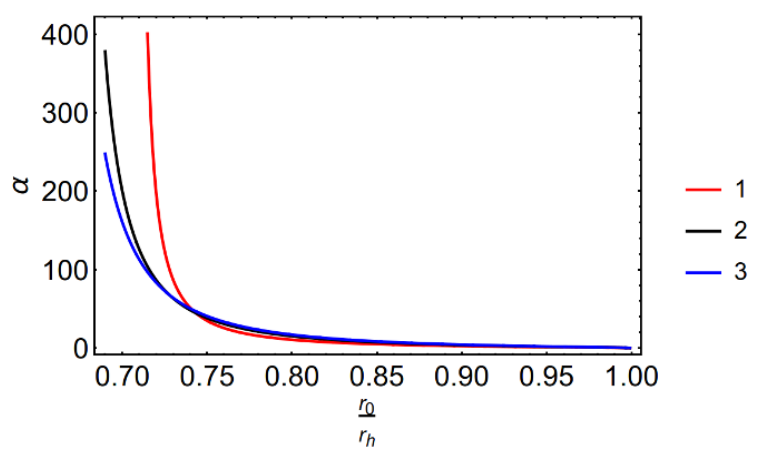

Figure 8. $\alpha$ as a function of $\frac{r_{0}}{r_{*}}$ at any temperature.
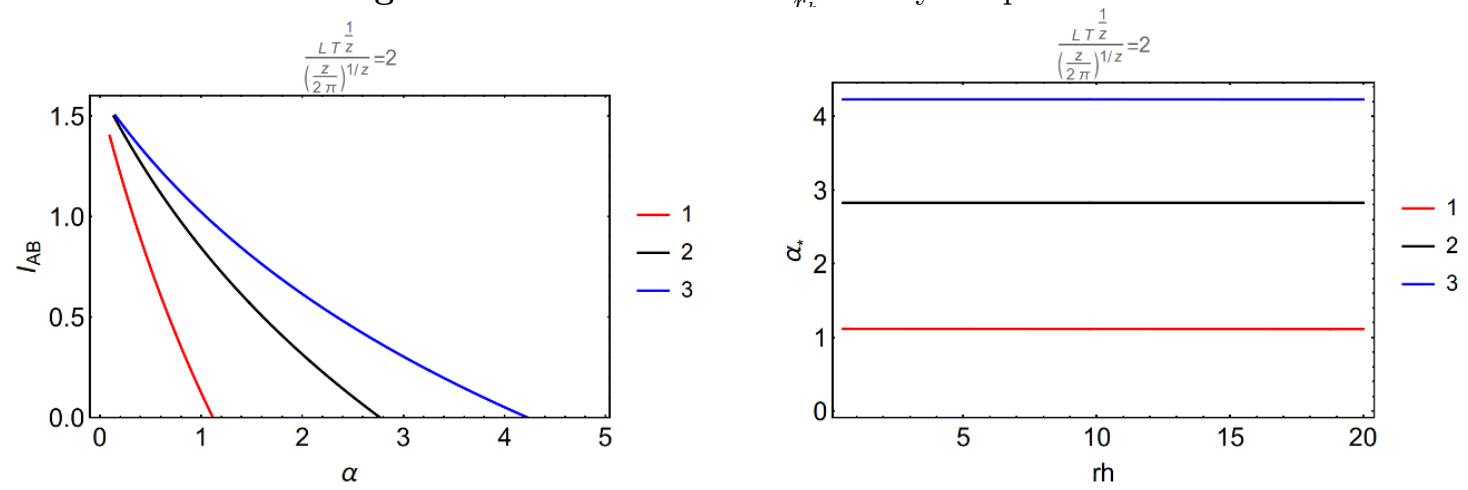

Figure 9. Left: $I(A ; B)$ as a function of $\alpha$ for various $z$ for $\frac{L T^{\frac{1}{z}}}{\left(\frac{z}{2 \pi}\right)^{\frac{1}{z}}}=2$. Right: $\alpha_{*}$ as a function of horizon radius, in units of $\ell$.

The entanglement entropy is a function of $r_{0}$, which can be related to $\alpha$, the shift in the shock wave geometry, similarly to Equation (3.36). Figure 8 shows a numerical plots of dependence of $\alpha$ on $\frac{r_{0}}{r_{h}}$ for various $z$. We can show that this behavior is independent of temperature for $\left(\frac{\Lambda}{r_{h}}\right)^{1 / z} \gg 1$.

\subsection{Mutual information in shock wave Lifshitz Black Hole}

We now calculate mutual information in the Lifshitz Black Hole in presence of a shock wave, for two-strip like regions defined in section 4.2. We follow the calculation in section 3.5. Figure 9 shows the behavior of mutual information for various $z$ as function of $\alpha$ for a fixed $L$ in units of temperature (i.e. fixed $\left.L(2 \pi T / z)^{1 / z}\right) . \alpha_{*}$ gives the value of $\alpha$ for which the mutual information vanishes. We can easily notice that $\alpha_{*}$ increases with $z$ for length of the boundary strip fixed in units of temperature. Also $\alpha_{*}$ is independent of $r_{h}$ or temperature for a given $z$ and length of the boundary strip fixed in units of temperature. Figure 10 shows variation of $\alpha_{*}$ with the length of the boundary strip fixed in units of temperature.

\section{Gauss-Bonnet gravity}

\subsection{Black hole geometry in Gauss-Bonnet gravity}

Finally, we would like to include in our analysis higher curvature corrections to the conformal case studied in [22]. Specifically, we focus on the planar black hole solution in 


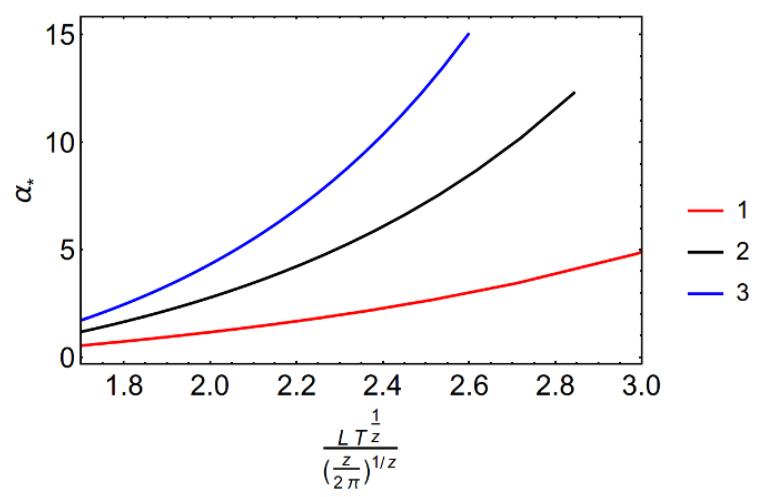

Figure 10. $\alpha_{*}$ as a function of length of the boundary strip fixed in units of temperature for various $z$.

Gauss-Bonnet gravity. The Gauss-Bonnet correction to the action for the 5-dimensional bulk in is given by $[30-32,34]$

$$
\begin{aligned}
S_{\text {grav }} & =\frac{1}{16 \pi G_{N}} \int d^{5} x \sqrt{-g}\left(R+\frac{12}{L^{2}}+\frac{\lambda_{\mathrm{GB}} L^{2}}{2} \mathcal{X}_{4}\right), \\
\mathcal{X}_{4} & =R_{\mu \nu \rho \sigma} R^{\mu \nu \rho \sigma}-4 R_{\mu \nu} R^{\mu \nu}+R^{2},
\end{aligned}
$$

where $R$ denotes the Ricci-scalar. The equations of motion that follow from this action are given by

$$
\begin{aligned}
& R_{\mu \nu}-\frac{1}{2} g_{\mu \nu}\left(R+\frac{12}{L^{2}}+\frac{\lambda_{\mathrm{GB}} L^{2}}{2} \mathcal{X}_{4}\right)+\lambda_{\mathrm{GB}} L^{2} \mathcal{H}_{\mu \nu}=0, \\
& \mathcal{H}=R_{\mu \rho \sigma \lambda} R_{\nu}{ }^{\rho \sigma \lambda}-2 R_{\mu \rho}{R_{\nu}}^{\rho}-2 R_{\mu \rho \nu \sigma} R^{\rho \sigma}+R R_{\mu \nu} .
\end{aligned}
$$

It can be checked that background metric

$$
\begin{aligned}
d s^{2} & =\frac{\ell^{2}}{z^{2}}\left[-f(z) d t^{2}+d \vec{x}^{2}+\frac{d z^{2}}{f(z)}\right], \\
f(z) & =\frac{1}{2 \lambda_{\mathrm{GB}} f_{0}}\left[1-\sqrt{1-4 \lambda_{\mathrm{GB}}\left(1-z^{4} / z_{h}^{4}\right)}\right], \\
f_{0} & \equiv \frac{1}{2 \lambda_{\mathrm{GB}}}\left(1-\sqrt{1-4 \lambda_{\mathrm{GB}}}\right), \quad \ell^{2} \equiv \frac{L^{2}}{f_{0}}
\end{aligned}
$$

obeys the equation of motion in (5.3) and that, in the $\lambda_{\mathrm{GB}} \rightarrow 0$ limit, we recover the AdSSchwarzschild background. Additionally, in this geometry, the singularity is at infinity for positive $\lambda_{\mathrm{GB}}$, and at $z=\frac{z_{h}}{\sqrt{2} \lambda_{\mathrm{GB}}^{1 / 4}}\left(4 \lambda_{\mathrm{GB}}-1\right)^{1 / 4}$.

The temperature in these coordinates is given by $1 / T=\beta=\pi f_{0} z_{h}$, and the entropy and energy densities are

$$
s=\frac{\ell^{3}}{4 G_{N} z_{h}^{3}}, \quad \mathcal{E}=\frac{3}{4} s T .
$$

Finally, the values of $\lambda_{\mathrm{GB}}$ were constrained by causality to the interval [37, 52]

$$
-7 / 36 \leq \lambda_{\mathrm{GB}} \leq 9 / 100 .
$$


However, more recently, it has been pointed out in [39] that Gauss-Bonnet gravity, taken as an exact theory, violates causality for any value of $\lambda_{\mathrm{GB}}$.

\subsection{Mutual information in Gauss-Bonnet gravity}

Our main purpose in this section is to examine how mutual information is disrupted for the a two-sided black hole given by (5.6). Following the prescription that we used for the non-conformal cases in section (3.2), we compute the mutual information between two intervals located in opposite sides of the black hole. We do this first for the unperturbed geometry and then for the metric that includes the backreaction of a shock wave coming from the left side of the hole.

The generalization of the Ryu-Takayanagi prescription to theories with Gauss-Bonnet gravity in the bulk has been proposed in [32, 33], building on [49]. ${ }^{6}$ Given a boundary region $A$ and a three dimensional surface $\Sigma$, whose boundary coincides with the two dimensional boundary $\partial A$, the entanglement entropy $S_{\mathrm{EE}}(A)$ is now obtained by extremizing

$$
S_{\mathrm{EE}}=\frac{1}{4 G_{N}} \int_{\Sigma} d^{3} \sigma \sqrt{\tilde{\gamma}}\left(1+\lambda_{\mathrm{GB}} f_{0} \ell^{2} R_{\Sigma}\right)+\frac{\lambda_{\mathrm{GB}} f_{0} \ell^{2}}{2 G_{N}} \int_{\partial \Sigma} d^{2} \sigma \sqrt{h} K,
$$

where $\sigma$ denotes the world-volume coordinates on $\Sigma$, and $\tilde{\gamma}$ is the induced metric on the surface. In addition, $R_{\Sigma}$ is the Ricci scalar corresponding to the induced metric on $\Sigma$ and the last term is the Gibbons-Hawking boundary term. ${ }^{7}$

Let us first compute the entanglement entropy $S_{A}$ (or $S_{B}$ ) for the "rectangular strip" $A, z=z(x), x \equiv x_{1} \in[-L / 2, L / 2], x_{2,3} \in(-\infty, \infty)$. With this parametrization, the induced metric on the surface is given by

$$
d s_{\text {ind }}^{2}=\frac{\ell^{2}}{z^{2}}\left(d x_{2}^{2}+d x_{3}^{2}\right)+\frac{\ell^{2}}{z^{2}}\left(1+\frac{z^{\prime 2}}{f}\right) d x^{2}, \quad ' \equiv d / d x .
$$

This yields the following effective area

$$
S_{\text {eff }}=\frac{\ell^{3} \mathcal{V}}{4 G_{N}} \int \frac{d x}{z^{3}}\left[\left(1+\frac{z^{\prime 2}}{f}\right)^{1 / 2}+2 \lambda_{\mathrm{GB}} \frac{z^{\prime 2}}{\left(1+\frac{z^{\prime 2}}{f}\right)^{1 / 2}}\right] .
$$

Since $x$ is a cyclic coordinate, its conjugated momentum is conserved. Thus, the equation of motion that minimizes the area is

$$
\frac{\sqrt{f}}{z^{3}} \frac{f+z^{\prime 2}-2 \lambda_{\mathrm{GB}} z^{\prime 2} f}{\left(f+z^{\prime 2}\right)^{3 / 2}}=\text { const }=\frac{1}{z_{\min }^{3}},
$$

where we have imposed the boundary condition that at $z=z_{\min }, z^{\prime}(x) \rightarrow 0$. A more convenient way to parametrize the minimal surface is by using $x(z)$ instead. In such a choice, we obtain the momentum conservation equation

$$
\sqrt{f} x^{\prime}(z)\left(\frac{1+f\left(x^{\prime 2}-2 \lambda_{\mathrm{GB}}\right)}{\left(1+f x^{\prime 2}\right)^{3 / 2}}\right)=\left(\frac{z}{z_{\min }}\right)^{3} .
$$

\footnotetext{
${ }^{6}$ The prescription for entanglement entropy in general theories with higher derivatives is given the Wald's entropy formula plus a term involving the extrinsic curvature [50, 51].

${ }^{7}$ The holographic EE in a time dependent Gauss-Bonnet gravity background was studied recently in [38].
} 


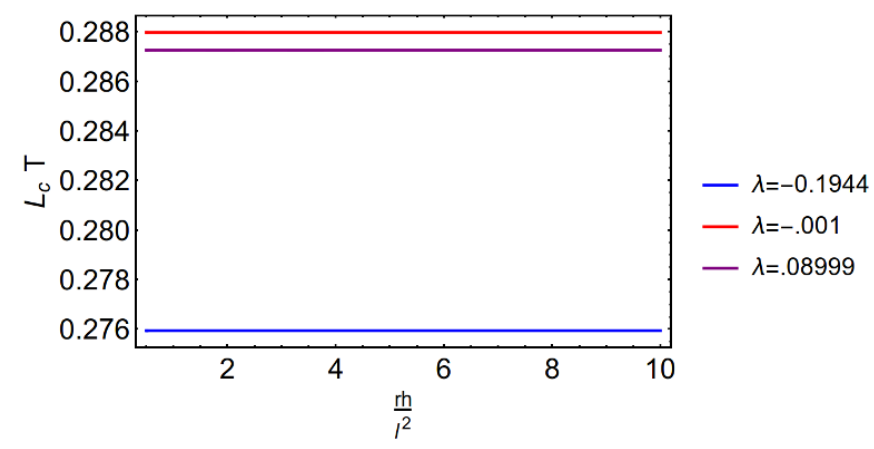

Figure 11. Critical values of $L$ as a function of the horizon $r_{H} \equiv \ell^{2} / z_{h}$, in units of $\ell$, for $\lambda_{\mathrm{GB}}=$ -0.1944 (blue), -0.0001(red), and 0.08999 (purple).

By defining the rescaled coordinate $\tau \equiv z / z_{*}$ and $\zeta(\tau) \equiv x^{\prime 2}$, we obtain the width of the intervals $A$ and $B$

$$
L=2 \int_{0}^{1} d \tau z_{\min } \sqrt{\zeta(\tau)}
$$

and the entanglement entropy is given by

$$
S_{A}=S_{B}=\frac{\ell^{3} \mathcal{V}}{2 G} \int_{\epsilon}^{1} d \tau \frac{z_{\min }^{-2}}{\tau^{3}}\left((1 / f+\zeta)^{1 / 2}+\frac{2 \lambda_{\mathrm{GB}}}{(1 / f+\zeta)^{1 / 2}}\right)
$$

On the other hand, the entanglement entropy $S_{A \cup B}$ is given by four times the area of the surface that extends from $(z, x)=(0, L / 2)$ (or equivalently $(0,0)$ due to translation symmetry) to the horizon, $(z, x)=\left(z_{h}, L / 2\right)$. This results in

$$
S_{A \cup B}=\frac{\ell^{3} \mathcal{V}}{G} \int_{\epsilon}^{z_{h} / z_{\min }} d \tau \frac{z_{\min }^{-2}}{\tau^{3}}\left(1 / \sqrt{f}+2 \lambda_{\mathrm{GB}} \sqrt{f}\right) .
$$

Thus, the mutual information between the intervals is given by

$$
I(A, B)=\frac{\ell^{3} \mathcal{V}}{G}\left\{\begin{array}{c}
\int_{\epsilon}^{1} d \tau \frac{z_{\min }^{-2}}{\tau^{3}}\left((1 / f+\zeta)^{1 / 2}+\frac{2 \lambda_{\mathrm{GB}}}{(1 / f+\zeta)^{1 / 2}}\right) \\
-\int_{\epsilon}^{\frac{z_{h}}{z_{\min }}} d \tau \frac{z_{\min }^{-2}}{\tau^{3}}\left(1 / \sqrt{f}+2 \lambda_{\mathrm{GB}} \sqrt{f}\right), \text { if }>0 \\
0, \quad \text { otherwise }
\end{array}\right.
$$

For low values of $z_{\text {min }}$ (i.e. small width $L$ ), the mutual information vanishes since the sum of the individual entanglement entropies, $S_{A}$ and $S_{B}$, is smaller than $S_{A \cup B}$. However, as $z_{\text {min }}$ approaches the horizon $z_{h}, I(A, B)$ becomes non-zero and is given by the first line in (5.17). Figure 11 shows the values of the interval width for which mutual information vanishes, for different values of $\lambda_{\mathrm{GB}}$.

\subsection{Shock wave geometry and scrambling time}

Let us now estimate the scrambling time by analyzing the perturbed geometry when a null shock wave is added from the left boundary (see figure 3). First, for the sake of comparison 
with previous sections, let us define $r \equiv \ell^{2} / z$ and rewrite (5.6) as

$$
d s^{2}=-g(r) d t^{2}+\frac{d r^{2}}{g(r)}+\frac{r^{2}}{\ell^{2}} d \vec{x}^{2}
$$

where now

$$
g(r) \equiv \frac{r^{2}}{\ell^{2} 2 \lambda_{\mathrm{GB}} f_{0}}\left(1-\sqrt{1-4 \lambda_{\mathrm{GB}}\left(1-\frac{r_{H}^{4}}{r^{4}}\right)}\right) .
$$

The temperature is given by

$$
\beta \equiv \frac{4 \pi}{g^{\prime}\left(r_{H}\right)}=\frac{f_{0} \ell^{2} \pi}{r_{H}}
$$

which depends on $\lambda_{\mathrm{GB}}$ through $f_{0}$ (see (5.6)).

The tortoise coordinate and the Kruskal coordinates are defined in the standard way. The backreaction of the shock wave into the geometry can be approximated again by a shift in the Kruskal time $v \rightarrow v+\alpha$. The tortoise coordinate $r_{*}$ is found to be

$$
\begin{aligned}
r_{*} & \approx \frac{f_{0} \ell^{2}}{4 r_{H}}\left(\log \left(\frac{r-r_{H}}{2 r_{H}}\right)-\pi / 2+4 \lambda_{\mathrm{GB}}\right)+\mathcal{O}\left(r-r_{H}\right) \\
& \approx \frac{\beta}{4 \pi}\left(\log \left(r-r_{H}\right)+C_{\lambda_{\mathrm{GB}}}\right), \quad C_{\lambda_{\mathrm{GB}}}=4 \lambda_{\mathrm{GB}}-\pi / 2-\log 2 r_{H} .
\end{aligned}
$$

As in the previous section, the scrambling time is then estimated by setting $\alpha \approx \mathcal{O}(1)$, which leads to

$$
t_{*}=\frac{\beta}{2 \pi} \log \frac{e^{-C_{\lambda_{\mathrm{GB}}}}}{\delta r_{H}} \approx \frac{\beta}{2 \pi} \log S_{\mathrm{BH}}+\frac{\beta}{2 \pi}\left(\frac{\pi}{2}-4 \lambda_{\mathrm{GB}}\right),
$$

for small values of $\left|\lambda_{\mathrm{GB}}\right|$.

In the next subsection, we will compute numerically the evolution of mutual information as a function of $\alpha$ to confirm that, indeed, it vanishes as $\alpha$ approaches values of order $\mathcal{O}(1)$.

\subsection{Disruption of mutual information after the shock wave}

Now we want to find the extremal surface that interpolates between the half plane on one side $\left(A_{h}\right)$ to the other side $\left(B_{h}\right)$ of the two-sided geometry. We use as (5.6) as the bulk metric. Similar to previous sections, we parameterize the surface by $z(t)$, in which case the induced surface is

$$
d s_{\text {ind }}^{2}=\frac{\ell^{2}}{z(t)^{2}}\left(d x_{2}^{2}+d x_{3}^{2}\right)+\frac{\ell^{2}}{z(t)^{2}}\left(-f+\frac{\dot{z}^{2}}{f}\right) d t^{2},
$$

and the effective action to be extremized is

$$
S_{\mathrm{eff}}=\frac{\ell^{3} \mathcal{V}}{4 G_{N}} \int \frac{d t}{z^{3}}\left[\left(-f+\frac{\dot{z}^{2}}{f}\right)^{1 / 2}+2 \lambda_{\mathrm{GB}} \frac{\dot{z}^{2}}{\left(-f+\frac{\dot{z}^{2}}{f}\right)^{1 / 2}}\right]
$$


Now the conserved quantity corresponding to the $t$-translation invariance is

$$
\gamma \equiv \frac{\sqrt{-f\left(z_{0}\right)}}{z_{0}^{3}}=\frac{f^{2}-\dot{z}^{2}\left(1-2 \lambda_{\mathrm{GB}} f\right)}{z^{3}\left(-f+\frac{\dot{z}^{2}}{f}\right)^{3 / 2}},\left.\quad \dot{z}\right|_{z_{0}}=0 .
$$

Using $t(z)$ to parameterize the surface, and defining $\eta(z) \equiv t^{\prime}(z)^{2}$, we obtain

$$
t(z)=\int_{0}^{z} d \tilde{z} \sqrt{\eta(\tilde{z})}
$$

where $\eta(z)$ is obtained from the real solution to the equation

$$
\gamma=\frac{\left(\eta f^{2}-\left(1-2 \lambda_{\mathrm{GB}} f\right)\right) \sqrt{\eta}}{z^{3}\left(-\eta f+\frac{1}{f}\right)^{3 / 2}}
$$

In order to find a relation between $\alpha$ and $z_{0}$, we divide the extremal surface in three sections, in the same way as explained in section 3.4 , using $d z_{*} \equiv-d z / f(z)$. In this case, we obtain

$$
\begin{aligned}
\alpha & =2 e^{K_{1}+K_{2}+K_{3}} \\
K_{1} & =-\frac{4 \pi}{\beta} \int_{z_{0}}^{\bar{z}} d z \frac{1}{-f(z)}, \\
K_{2} & =\frac{2 \pi}{\beta} \int_{0}^{z_{h}} d z\left(\frac{1}{-f(z)}-\sqrt{\eta(z)}\right), \\
K_{3} & =\frac{4 \pi}{\beta} \int_{z h}^{z_{0}} d z\left(\frac{1}{-f(z)}-\sqrt{\eta(z)}\right),
\end{aligned}
$$

where $\bar{z}$ is, again, a surface inside the black hole where $z_{*}=0$.

Figure 12 depicts $\alpha$ as a function of the "turning" point $z_{0}$. In the case of positive $\lambda_{\mathrm{GB}}$ (green line), we see that the rapid growth is enhanced and qualitatively does not differ from the gravity calculations Einstein. However, a drastically different behavior is found for negative values of the Gauss-Bonnet coupling. In that case, $\alpha$ reaches a maximum value and then decreases until it reaches a critical value. This last value corresponds to the fact that $z_{0}$ has reached the singularity. We will see what this implies for EE and mutual information in the next paragraphs.

The entanglement entropy that corresponds to the extremal surface described above is given by

$$
\begin{aligned}
S_{A_{h} \cup B_{h}}=\frac{\ell^{3} \mathcal{V}}{G_{N}}\left[\int_{0}^{z_{h}} d z\right. & \frac{1}{z^{3}}\left((-f \eta+1 / f)^{1 / 2}+\frac{2 \lambda_{\mathrm{GB}}}{(-f \eta+1 / f)^{1 / 2}}\right) \\
& \left.+2 \int_{z_{h}}^{z_{0}} d z \frac{1}{z^{3}}\left((-f \eta+1 / f)^{1 / 2}+\frac{2 \lambda_{\mathrm{GB}}}{(-f \eta+1 / f)^{1 / 2}}\right)\right] .
\end{aligned}
$$

In figure 13 we show mutual information as a function of $\alpha$, which is given now obtained by subtracting (5.30) from twice the result of (5.15). For positive $\lambda_{\mathrm{GB}}$, the behavior is 


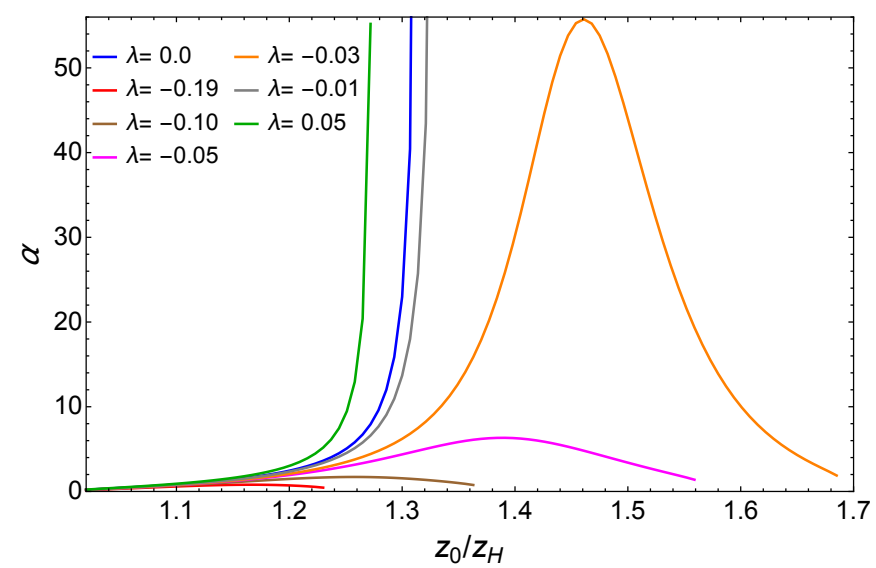

Figure 12. Values of $\alpha$ as a function of $z_{0}$, for several values of $\lambda_{\mathrm{GB}}$.

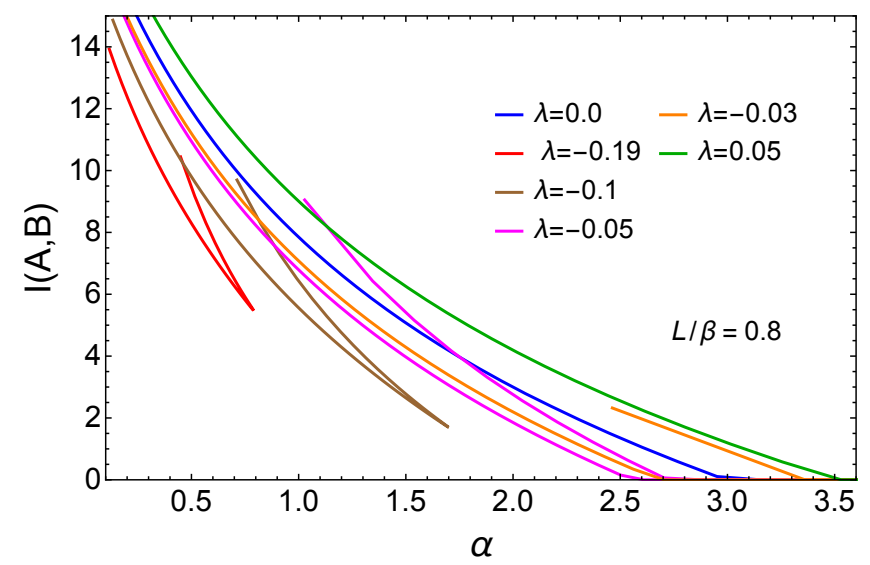

Figure 13. Mutual information $I(A, B)$ as a function of $\alpha$ for several values of $\lambda_{\mathrm{GB}}$. In these plots, we used $L / \beta=0.8$.

similar to the case of no Gauss-Bonnet coupling, and mutual information indeed vanishes for $\alpha \sim \mathcal{O}(1)$ as assumed in the calculation of the scrambling time in section 5.3. For negative $\lambda_{\mathrm{GB}}$, however, we notice here an anomalous behavior, due to the existence of two values of $z_{0}$ for a given value of $\alpha$ (see figure 12). Following the prescription from [3] to compute $S_{A_{h} \cup B_{h}}$, we must choose the surface with smaller area. This implies a "jump" in the evolution of entanglement entropy and mutual information as the dependence between the two sides is erased. Likewise, there is a second discontinuity at the end of scrambling which happens when the disconnected regions at each side of the eternal black black hole have a greater area than the surface that interpolates between the two boundaries. This is further illustrated in figure 14. It is worth noticing that a similar behavior was also found in the analysis of EE in a Vaidya-like background in [38].

\section{Outlook}

In the context of generalized holography (not restricted to asymptotically AdS space times), we have shown that the dual thermal field theory scrambles information at time scales given 


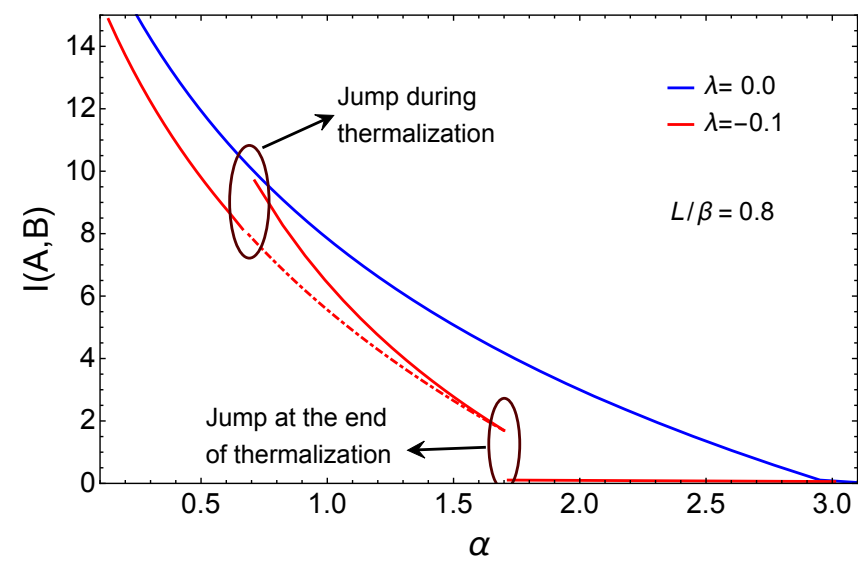

Figure 14. Discontinuities in mutual information, $I(A, B)$, as a function of $\alpha$ for negative $\lambda_{\mathrm{GB}}$ (solid red line). The blue line shows the case of no Gauss-Bonnet coupling for the sake of comparison.

by $\frac{\beta}{2 \pi} \log S$. There are in general some sub-leading corrections which depend on the various parameters of the bulk geometry. The scrambling behavior was studied (holographically) by following time dependence of thermo-mutual information for some given region in the boundary theory. For non-conformal and non-relativistic cases, the behavior was found to be similar to the conformal case with $a=c$. In case of theories dual to Gauss Bonnet Gravity, corresponding in particular to the conformal case with $c \neq a$, the behavior is very different depending on the sign of Gauss Bonnet coupling. The results were summarized in the introduction to this article. We would like to conclude by mentioning some of the open issues to which we want to return in future work.

- Thermo-mutual information [18], studied here as a probe of scrambling, involves regions in two different field theories of the thermofield double. It will be interesting to understand the map of thermo-mutual information to a probe defined only on one side, generalizing the map in case of $1+1$ dimensional CFT, studied in [18].

- The scrambling time $\left(t_{*}\right)$ is defined by considering vanishing of this thermo-mutual information [16]. Alternatively scrambling time $\left(\tilde{t}_{*}\right)$ is also defined by exponential fall of a function $F(t)$ [26] defined in terms of four point functions in the field theory. In the case of the BTZ black hole and its dual theory, it can be shown [16] that the two scrambling times are essentially the same, at least in case of some heavy operators. However, a precise connection between the two is still missing in the literature.

- In the alternate probe of scrambling in terms of four point functions, the Lyapunov exponent provides the rate of exponential decrease of $F(t)$ at early times [26]; whereas the late-time decay is characterized by the poles of the Green's function, which holographically correspond to quasinormal modes. In [26], a bound on the Lyapunov exponent was also proposed. A precise definition of the Lyapunov exponent in terms of the behavior of mutual information would be very useful. 
- In this note, we considered the Gauss Bonnet higher curvature gravitational theory. An interesting question is obviously how would the mutual information behave in the cases of other higher curvature corrections to the gravitational action.

\section{Acknowledgments}

We would like to thank specially to S. Yankielowicz for very productive and encouraging discussions. We are grateful to O. Aharony, O. Ben-Ami, E. Cáceres, D. Carmi, G. Gur-Ari, D. Harlow, N. Itzhaki, V. Kaplunovsky, L. Pando Zayas, and V. Rosenhaus, for insightful conversations and comments. This work was supported in part by a centre of excellence supported by the Israel Science Foundation (grant number 1989/14), and by the US-Israel bi-national fund (BSF) grant number 2012383 and the Germany Israel bi-national fund GIF grant number I-244-303.7-2013. The work of N.S was supported by "The PBC program for fellowships for outstanding post-doctoral researcher from China and India of the Israel council of higher education".

\section{A A series expansion of EE in $D p$-brane backgrounds}

In this appendix we include a series approximation of the entanglement entropy of one strip, as a supplement to the numerical calculation of section 3.2. There, we obtained that $S_{A}$ or $S_{B}$, with width $L$, is given by the extremized area

$$
\text { Area }_{d-2}=2 \frac{\mathcal{V}}{\ell^{d-1}} \ell^{8-p-\sigma} \int_{r_{\min }}^{\infty} d r \frac{r^{\sigma}}{\sqrt{1-\frac{r_{H}^{s}}{r^{s}}}} \frac{1}{\sqrt{1-\left(\frac{r_{\min }}{r}\right)^{\omega}}}
$$

where

$$
\begin{aligned}
\sigma & \equiv \frac{n}{16}(7-p)^{2}(p-2)+\frac{n}{16}(p-3)^{2}(8-p), \quad s \equiv n(7-p), \\
\omega & \equiv \frac{p n}{8}(7-p)^{2}+\frac{n}{8}(p-3)^{2}(8-p),
\end{aligned}
$$

and $r=\Lambda$ is set as the cutoff. The divergent part of the area yields

$$
\text { Area }_{\mathrm{div}}=2 \frac{\mathcal{V}}{\ell^{d-1}} \ell^{8-p-\sigma} \frac{\Lambda^{\sigma+1}}{\sigma+1}
$$

and the width $L$ is given by

$$
L=2 \int_{r_{\min }}^{\infty} d r\left(\frac{r}{\ell}\right)^{-2 \xi} \frac{1}{\sqrt{1-\frac{r_{H}^{s}}{r^{s}}} \sqrt{\left(\frac{r}{r_{\min }}\right)^{\omega}-1}} .
$$

Using the coordinate redefinition $u \equiv \frac{r_{\min }}{r}$, we rewrite the area as

$$
\text { Area }_{d-2}^{\text {finite }}=2 \frac{\mathcal{V}}{\ell^{d-1}} \ell^{8-p-\sigma} r_{\min }^{1+\sigma} \int_{r_{\min } / \Lambda}^{1} d u \frac{u^{-2-\sigma}}{\sqrt{1-u^{\omega}}} \frac{1}{\sqrt{1-\left(\frac{r_{H}}{r_{\min }} u\right)^{s}}}-\text { Area }_{\mathrm{div}}
$$


and the lengths as

$$
L=2 \ell^{2 \xi} r_{\min }^{1-2 \xi} \int_{0}^{1} d u u^{-2+4 \gamma+\sigma} \frac{1}{\sqrt{1-u^{\omega}} \sqrt{1-\frac{r_{H}^{s}}{r_{\min }^{s}} u^{s}}} .
$$

Now we would like to have some analytic approximation for these results. To do so, we use the fact that $r_{\min }>r_{H}$, and perform the series expansion [46]

$$
\frac{1}{\sqrt{1-\left(\frac{r_{H}}{r_{\min }} u\right)^{s}}}=\sum_{n=0}^{\infty} \frac{\Gamma[n+1 / 2]}{\sqrt{\pi} \Gamma[n+1]}\left(\frac{r_{H}}{r_{\min }}\right)^{n s} u^{n s}
$$

which results in

$$
\begin{aligned}
\text { Area }_{d-2}^{\text {finite }}=2 \frac{\mathcal{V}}{\ell^{d-1}} \ell^{8-p-\sigma} r_{\min }^{1+\sigma} & {\left[-\frac{\sqrt{\pi} \Gamma\left[1-\frac{1+\sigma}{\omega}\right]}{(1+\sigma) \Gamma\left[\frac{1}{2}-\frac{1+\sigma}{\omega}\right]}\right.} \\
& \left.+\sum_{n=1}^{\infty} \frac{\Gamma[n+1 / 2] \Gamma\left[\frac{n s-1-\sigma}{\omega}\right]}{\omega \Gamma[n+1] \Gamma\left[\frac{2 n s-2-2 \sigma+\omega}{2 \omega}\right]}\left(\frac{r_{H}}{r_{\min }}\right)^{n s}\right],
\end{aligned}
$$

and

$$
L=2 \ell^{2 \xi} r_{\min }^{1-2 \xi} \sum_{n=0}^{\infty} \frac{\Gamma[n+1 / 2] \Gamma\left[\frac{n s-1+4 \xi-\sigma}{\omega}\right]}{\omega \Gamma[n+1] \Gamma\left[\frac{2 n s-2+2(4 \xi-\sigma)+\omega}{2 \omega}\right]}\left(\frac{r_{H}}{r_{\min }}\right)^{n s} .
$$

Both expressions are convergent and finite for the case $r_{\min } \gg r_{H}$. But we are interested in the case where $r_{\min } \sim r_{H}$, since this is the regime where mutual information will become very small. In order to obtain a convergent expression, we need to reorganize (A.8). Using the properties of the gamma function we can rewrite the coefficients of the sum in (A.8) and write the area as

$$
\begin{array}{r}
\text { Area }_{d-2}^{\text {finite }}=2 \frac{\mathcal{V}}{\ell^{d-1}} \ell^{8-p-\sigma} \\
r_{\min }^{1+\sigma}\left[\left(\frac{r_{\min }}{\ell}\right)^{2 \xi} \frac{L}{2 r_{\min }}-\frac{\sqrt{\pi}(\omega-2 \sigma) \Gamma\left[1-\frac{1+\sigma}{\omega}\right]}{\omega \Gamma\left[\frac{3 \omega-2(\sigma+1)}{2 \omega}\right]}\right. \\
\left.+\sum_{n=1}^{\infty} \frac{1}{n s+(\sigma+1)} \frac{\Gamma[n+1 / 2] \Gamma\left[\frac{n s-1-\sigma}{\omega}\right]}{\Gamma[n+1] \Gamma\left[\frac{2 n s-2-2 \sigma+\omega}{2 \omega}\right]}\left(\frac{r_{H}}{r_{\min }}\right)^{n s}\right],
\end{array}
$$

which is now convergent for in the limit $r_{\min } \sim r_{H}$. In such a limit, we obtain

$$
\begin{aligned}
\text { Area }_{d-2}^{\text {finite }} & =2 \frac{\mathcal{V}}{\ell^{d-1}} \ell^{8-p-\sigma} r_{H}^{1+\sigma}\left(\left(\frac{r_{H}}{\ell}\right)^{2 \gamma} \frac{L}{2 r_{H}}+\mathcal{A}_{1}\right), \\
\mathcal{A}_{1} & \equiv-\frac{\sqrt{\pi}(\omega-2 \sigma) \Gamma\left[1-\frac{1+\sigma}{\omega}\right]}{\omega \Gamma\left[\frac{3 \omega-2(\sigma+1)}{2 \omega}\right]}+\sum_{n=1}^{\infty} \frac{1}{n s+(\sigma+1)} \frac{\Gamma[n+1 / 2] \Gamma\left[\frac{n s-1-\sigma}{\omega}\right]}{\Gamma[n+1] \Gamma\left[\frac{2 n s-2-2 \sigma+\omega}{2 \omega}\right]} .
\end{aligned}
$$

Now, we compute the area of the surface that interpolates between the two boundaries. This area corresponds to four times the area of a surface that divides the boundary in half 
and extends from one boundary to the black hole. Defining $\tilde{u} \equiv u / r_{H}$,

$$
\begin{aligned}
\text { Area }_{A \cup B} & =4 \frac{\mathcal{V}}{\ell^{d-1}} \ell^{8-p-\sigma} r_{H}^{\sigma+1} \int_{r_{H} / \Lambda}^{1} d \tilde{u} \frac{\tilde{u}^{-2-\sigma}}{\sqrt{1-\tilde{u}^{s}}}-2 \text { Area }_{\mathrm{div}}, \\
& =4 \frac{\mathcal{V}}{\ell^{d-1}} \ell^{8-p-\sigma} r_{H}^{\sigma+1} \frac{\sqrt{\pi} \Gamma\left[-\frac{\sigma+1}{s}\right]}{s \Gamma\left[\frac{s-2 \sigma-2}{2 s}\right]} \equiv 4 \frac{\mathcal{V}}{\ell^{d-1}} \ell^{8-p-\sigma} r_{H}^{\sigma+1} \mathcal{A}_{2}
\end{aligned}
$$

Thus, the mutual information between $A$ and $B$ is given by

$$
I(A, B) \equiv S_{A}+S_{B}-S_{A \cup B}=\frac{\mathcal{V}}{\ell^{d-1} G_{N}} \ell^{8-p-\sigma} r_{H}^{\sigma+1}\left(\left(\frac{r_{H}}{\ell}\right)^{2 \xi} \frac{L}{2 r_{H}}+\mathcal{A}_{1}-\mathcal{A}_{2}\right) .
$$

\section{B Two sided entanglement entropy for half plane in the case of D5 brane}

Let us consider the case with $p=5$, i.e. $D 5$ brane. We can repeat the calculations in section (3.4) in this case, but now analytically. We have used these results to do a consistancy check on our numerics. Let us define $\tilde{\gamma}^{2}=\frac{2 R \gamma^{2}}{r_{0}^{8}}=\frac{r_{h}^{4}}{r_{0}^{4}}-1 \geq 0$ (as $r_{0}<r_{h}$ ). The inverse temperature is given by $\beta=4 \pi \ell$ (note that it does not depend on the horizon radius). We have used this analytical results as a reference for our numerical calculations. We can get an analytic solution for minimal surface as,

$$
t\left(x=\frac{r}{r_{h}}\right)=\ell \log \left(\left|x^{4}-1\right|\right)-\ell \log \left|x^{4}-1+2 \tilde{\gamma}^{2}+\tilde{\gamma} \sqrt{4 \tilde{\gamma}^{2}+x^{4}\left(x^{4}-1\right)}\right| .
$$

Also,

$$
r_{*}\left(x=\frac{r}{r_{h}}\right)=\int \frac{d r}{f(r)}=\ell \log \left(\left|x^{4}-1\right|\right) .
$$

We will choose $\bar{x}=\frac{\bar{r}}{r_{h}}=0$ in the following calculations. The various integrals $\left(x_{0}=\frac{r_{0}}{r_{h}}\right.$, $\left.x_{\Lambda}=\frac{r_{\Lambda}}{r_{h}}\right)$,

$$
\begin{aligned}
& K_{1}=\log \left(1-x_{0}^{4}\right) \\
& K_{2} \simeq 2 \log x_{\Lambda}+\frac{1}{2} \log \left(\frac{1+2 x_{0}^{2} \sqrt{1-x_{0}^{4}}}{4 x_{0}^{4}\left(1-x_{0}^{4}\right)}\right), \quad x_{\Lambda} \gg 1, \\
& K_{3}=\log \left(\frac{4 x_{0}^{4}}{2 x_{0}^{4}-1}\right), \\
& u_{\Lambda} \simeq x_{\Lambda}^{2}, \quad x_{\Lambda} \gg 1 .
\end{aligned}
$$

Then,

$$
\alpha=\frac{4 x_{0}^{2} \sqrt{1-x_{0}^{4}} \sqrt{1+2 x_{0}^{2} \sqrt{1-x_{0}^{4}}}}{2 x_{0}^{4}-1} .
$$

This equations clearly shows that there is critical value of $x_{0}=x_{\text {crit }}=2^{-\frac{1}{4}}$ beyond which surface does not penetrate the horizon.

We can invert this relation to get $x_{0}$ in terms of $\alpha$,

$$
x_{0}^{2}=\frac{1}{8}\left(-\alpha+\sqrt{16+\alpha^{2}}+\sqrt{16-2 \alpha^{2}+2 \alpha \sqrt{16+\alpha^{2}}}\right) .
$$


We can now calculate the area for this surface which will correspond to $S_{A \cup B}$. This has two ultraviolet $\alpha$ independent divergence $\left(\sim \Lambda^{4}, \quad \log (\Lambda)\right)$ piece. The regularized entanglement entropy (defined in (3.38)) is given by,

$$
S_{A_{h} \cup B_{h}}^{\mathrm{reg}}(\alpha)=-n_{5}\left[\frac{1}{2} \alpha\left(\alpha-\sqrt{16+\alpha^{2}}\right)-8 \log \left(\alpha+\sqrt{16+\alpha^{2}}\right)+16 \log 2\right],
$$

where $n_{5}=\frac{1}{64} \frac{\mathcal{V} r_{h}^{4}}{4 G_{N}}$. The regularized entanglement entropy for large $\alpha$ grows as $\log \alpha$.

Open Access. This article is distributed under the terms of the Creative Commons Attribution License (CC-BY 4.0), which permits any use, distribution and reproduction in any medium, provided the original author(s) and source are credited.

\section{References}

[1] S. Ryu and T. Takayanagi, Holographic derivation of entanglement entropy from AdS/CFT, Phys. Rev. Lett. 96 (2006) 181602 [hep-th/0603001] [InSPIRE].

[2] S. Ryu and T. Takayanagi, Aspects of holographic entanglement entropy, JHEP 08 (2006) 045 [hep-th/0605073] [INSPIRE].

[3] V.E. Hubeny, M. Rangamani and T. Takayanagi, A covariant holographic entanglement entropy proposal, JHEP 07 (2007) 062 [arXiv:0705.0016] [INSPIRE].

[4] G.T. Horowitz and V.E. Hubeny, Quasinormal modes of AdS black holes and the approach to thermal equilibrium, Phys. Rev. D 62 (2000) 024027 [hep-th/9909056] [INSPIRE].

[5] V. Balasubramanian et al., Holographic thermalization, Phys. Rev. D 84 (2011) 026010 [arXiv: 1103.2683] [INSPIRE].

[6] A. Allais and E. Tonni, Holographic evolution of the mutual information, JHEP 01 (2012) 102 [arXiv: 1110.1607] [INSPIRE].

[7] R. Callan, J.-Y. He and M. Headrick, Strong subadditivity and the covariant holographic entanglement entropy formula, JHEP 06 (2012) 081 [arXiv: 1204.2309] [INSPIRE].

[8] D. Galante and M. Schvellinger, Thermalization with a chemical potential from AdS spaces, JHEP 07 (2012) 096 [arXiv: 1205.1548] [INSPIRE].

[9] E. Caceres and A. Kundu, Holographic thermalization with chemical potential, JHEP 09 (2012) 055 [arXiv: 1205.2354] [INSPIRE].

[10] E. Caceres, A. Kundu, J.F. Pedraza and W. Tangarife, Strong subadditivity, null energy condition and charged black holes, JHEP 01 (2014) 084 [arXiv:1304.3398] [INSPIRE].

[11] H. Liu and S.J. Suh, Entanglement tsunami: universal scaling in holographic thermalization, Phys. Rev. Lett. 112 (2014) 011601 [arXiv: 1305.7244] [INSPIRE].

[12] H. Liu and S.J. Suh, Entanglement growth during thermalization in holographic systems, Phys. Rev. D 89 (2014) 066012 [arXiv:1311.1200] [INSPIRE].

[13] S. Kundu and J.F. Pedraza, Spread of entanglement for small subsystems in holographic CFTs, arXiv:1602.05934 [INSPIRE].

[14] P. Hayden and J. Preskill, Black holes as mirrors: quantum information in random subsystems, JHEP 09 (2007) 120 [arXiv:0708.4025] [INSPIRE]. 
[15] Y. Sekino and L. Susskind, Fast scramblers, JHEP 10 (2008) 065 [arXiv:0808.2096] [INSPIRE].

[16] S.H. Shenker and D. Stanford, Black holes and the butterfly effect, JHEP 03 (2014) 067 [arXiv:1306.0622] [INSPIRE].

[17] J.M. Maldacena, Eternal black holes in Anti-de Sitter, JHEP 04 (2003) 021 [hep-th/0106112] [INSPIRE].

[18] I.A. Morrison and M.M. Roberts, Mutual information between thermo-field doubles and disconnected holographic boundaries, JHEP 07 (2013) 081 [arXiv: 1211.2887] [INSPIRE].

[19] A. Fraser and H. Swinney, Independent coordinates for strange attractors from mutual information, Phys. Rev. A 33 (1986) 1134.

[20] P. Caputa, J. Simón, A. Štikonas, T. Takayanagi and K. Watanabe, Scrambling time from local perturbations of the eternal BTZ black hole, JHEP 08 (2015) 011 [arXiv:1503.08161] [INSPIRE].

[21] P. Hosur, X.-L. Qi, D.A. Roberts and B. Yoshida, Chaos in quantum channels, JHEP 02 (2016) 004 [arXiv: 1511.04021] [INSPIRE].

[22] S. Leichenauer, Disrupting entanglement of black holes, Phys. Rev. D 90 (2014) 046009 [arXiv: 1405.7365] [INSPIRE].

[23] S.H. Shenker and D. Stanford, Multiple shocks, JHEP 12 (2014) 046 [arXiv:1312.3296] [INSPIRE].

[24] D.A. Roberts, D. Stanford and L. Susskind, Localized shocks, JHEP 03 (2015) 051 [arXiv: 1409.8180] [INSPIRE].

[25] S.H. Shenker and D. Stanford, Stringy effects in scrambling, JHEP 05 (2015) 132 [arXiv: 1412.6087] [INSPIRE].

[26] J. Maldacena, S.H. Shenker and D. Stanford, A bound on chaos, arXiv:1503.01409 [INSPIRE].

[27] S. Kalyana Rama and B. Sathiapalan, On the role of chaos in the AdS/CFT connection, Mod. Phys. Lett. A 14 (1999) 2635 [hep-th/9905219] [INSPIRE].

[28] N. Itzhaki, J.M. Maldacena, J. Sonnenschein and S. Yankielowicz, Supergravity and the large- $N$ limit of theories with sixteen supercharges, Phys. Rev. D 58 (1998) 046004 [hep-th/9802042] [INSPIRE].

[29] K. Balasubramanian and J. McGreevy, An Analytic Lifshitz black hole, Phys. Rev. D 80 (2009) 104039 [arXiv:0909.0263] [INSPIRE].

[30] R.-G. Cai, Gauss-Bonnet black holes in AdS spaces, Phys. Rev. D 65 (2002) 084014 [hep-th/0109133] [INSPIRE].

[31] R.C. Myers and B. Robinson, Black holes in quasi-topological gravity, JHEP 08 (2010) 067 [arXiv: 1003.5357] [INSPIRE].

[32] J. de Boer, M. Kulaxizi and A. Parnachev, Holographic entanglement entropy in Lovelock gravities, JHEP 07 (2011) 109 [arXiv:1101.5781] [INSPIRE].

[33] L.-Y. Hung, R.C. Myers and M. Smolkin, On holographic entanglement entropy and higher curvature gravity, JHEP 04 (2011) 025 [arXiv:1101.5813] [INSPIRE]. 
[34] R.C. Myers and A. Singh, Comments on holographic entanglement entropy and RG flows, JHEP 04 (2012) 122 [arXiv: 1202.2068] [INSPIRE].

[35] J.L.F. Barbon and C.A. Fuertes, A note on the extensivity of the holographic entanglement entropy, JHEP 05 (2008) 053 [arXiv:0801.2153] [INSPIRE].

[36] U. Kol, C. Núñez, D. Schofield, J. Sonnenschein and M. Warschawski, Confinement, phase transitions and non-locality in the entanglement entropy, JHEP 06 (2014) 005 [arXiv: 1403.2721] [INSPIRE].

[37] M. Brigante, H. Liu, R.C. Myers, S. Shenker and S. Yaida, The viscosity bound and causality violation, Phys. Rev. Lett. 100 (2008) 191601 [arXiv:0802.3318] [INSPIRE].

[38] E. Caceres, M. Sanchez and J. Virrueta, Holographic entanglement entropy in time dependent Gauss-Bonnet gravity, arXiv:1512.05666 [INSPIRE].

[39] X.O. Camanho, J.D. Edelstein, J. Maldacena and A. Zhiboedov, Causality constraints on corrections to the graviton three-point coupling, JHEP 02 (2016) 020 [arXiv:1407.5597] [INSPIRE].

[40] I.R. Klebanov, D. Kutasov and A. Murugan, Entanglement as a probe of confinement, Nucl. Phys. B 796 (2008) 274 [arXiv:0709.2140] [INSPIRE].

[41] I. Bah, A. Faraggi, L.A. Pando Zayas and C.A. Terrero-Escalante, Holographic entanglement entropy and phase transitions at finite temperature, Int. J. Mod. Phys. A 24 (2009) 2703 [arXiv: 0710.5483] [INSPIRE].

[42] A. van Niekerk, Entanglement entropy in non-conformal holographic theories, arXiv:1108.2294 [INSPIRE].

[43] S. Kachru, X. Liu and M. Mulligan, Gravity duals of Lifshitz-like fixed points, Phys. Rev. D 78 (2008) 106005 [arXiv: 0808.1725] [INSPIRE].

[44] V. Keranen, E. Keski-Vakkuri and L. Thorlacius, Thermalization and entanglement following a non-relativistic holographic quench, Phys. Rev. D 85 (2012) 026005 [arXiv:1110.5035] [INSPIRE].

[45] H.J. Boonstra, K. Skenderis and P.K. Townsend, The domain wall/QFT correspondence, JHEP 01 (1999) 003 [hep-th/9807137] [INSPIRE].

[46] W. Fischler and S. Kundu, Strongly coupled gauge theories: high and low temperature behavior of non-local observables, JHEP 05 (2013) 098 [arXiv:1212.2643] [INSPIRE].

[47] G. 't Hooft, The scattering matrix approach for the quantum black hole: an overview, Int. J. Mod. Phys. A 11 (1996) 4623 [gr-qc/9607022] [INSPIRE].

[48] N. Itzhaki, Some remarks on 't Hooft's S matrix for black holes, hep-th/9603067 [INSPIRE].

[49] T. Jacobson and R.C. Myers, Black hole entropy and higher curvature interactions, Phys. Rev. Lett. 70 (1993) 3684 [hep-th/9305016] [INSPIRE].

[50] X. Dong, Holographic entanglement entropy for general higher derivative gravity, JHEP 01 (2014) 044 [arXiv: 1310.5713] [INSPIRE].

[51] J. Camps, Generalized entropy and higher derivative Gravity, JHEP 03 (2014) 070 [arXiv:1310.6659] [INSPIRE].

[52] A. Buchel, J. Escobedo, R.C. Myers, M.F. Paulos, A. Sinha and M. Smolkin, Holographic GB gravity in arbitrary dimensions, JHEP 03 (2010) 111 [arXiv:0911.4257] [INSPIRE]. 
[53] M. Taylor, Non-relativistic holography, arXiv:0812.0530 [INSPIRE].

[54] M. Taylor, Lifshitz holography, Class. Quant. Grav. 33 (2016) 033001 [arXiv:1512.03554] [INSPIRE].

[55] M. Alishahiha, A.F. Astaneh and M.R.M. Mozaffar, Thermalization in backgrounds with hyperscaling violating factor, Phys. Rev. D 90 (2014) 046004 [arXiv:1401.2807] [INSPIRE].

[56] P. Fonda, L. Franti, V. Keränen, E. Keski-Vakkuri, L. Thorlacius and E. Tonni, Holographic thermalization with Lifshitz scaling and hyperscaling violation, JHEP 08 (2014) 051 [arXiv: 1401.6088] [INSPIRE]. 